\title{
NUMERICAL ANALYSIS OF AN ELLIPTIC-PARABOLIC PARTIAL DIFFERENTIAL EQUATION*
}

\section{JOEL N. FRANKLIN† AND EUGENE R. RODEMICH $\ddagger$}

1. Introduction. G. Fichera [1] and other authors have investigated partial differential equations of the form

$$
\sum \sum a_{i j}(x) \frac{\partial^{2} u}{\partial x_{i} \partial x_{j}}+\sum a_{i}(x) \frac{\partial u}{\partial x_{i}}+a(x) u=f(x)
$$

in which the matrix $\left(a_{i j}(x)\right)$ is required to be positive semidefinite. Equations of this type occur in the theory of random processes. A numerical analysis of some equations of this type has been given by Cannon and Hill [9].

In this paper we consider a particular boundary value problem

$$
\frac{1}{2} \frac{\partial^{2} T}{\partial y^{2}}+y \frac{\partial T}{\partial x}=-1, \quad|x|<1, \quad|y|<\infty,
$$

where we require

$$
T(1, y)=0 \text { for } y>0, \quad T(-1, y)=0 \text { for } y<0,
$$

and

$$
T(x, y) \rightarrow 0 \text { as }|y| \rightarrow \infty .
$$

A problem of this sort was discussed analytically by W. Fleming [2], but he did not obtain an explicit solution for $T(x, 0)$.

The solution $T(x, y)$ is related to a randomly-accelerated particle whose position $\xi(t)$ satisfies the stochastic differential equation

$$
\frac{d^{2} \xi}{d t^{2}}=w(t), \quad t>0
$$

where $w(t)$ is white Gaussian noise. If the initial position and velocity are $\xi(0)=x$ and $\xi^{\prime}(0)=y$, where $|x|<1$, then $T(x, y)$ is the expected value of the first time at which the position $\xi(t)$ equals \pm 1 .

We obtain an analytic solution for $T(x, y)$ in terms of hypergeometric functions and confluent hypergeometric functions. We use this analytic solution to test the validity of numerical methods which are applicable to

* Received by the editors May 23, 1968.

$\uparrow$ Booth Computing Center, California Institute of Technology, Pasadena, California 91109.

‡ Jet Propulsion Laboratory, California Institute of Technology, Pasadena, California 91109. The work of this author was supported by the National Aeronautics and Space Administration. 
general elliptic-parabolic equations (1.1). We show that, even though the truncation error for the difference equations does not tend to zero, nevertheless the difference methods give convergence to the true solution. Sample calculations indicate the rates of convergence of the difference methods.

Each difference method requires the solution of a large number of simultaneous linear difference equations. We give iterative methods for solving these equations, and we prove that the iterations converge.

2. Derivation of the boundary value problem. Because the boundary value problems arising in the theory of stochastic processes are relatively unfamiliar to numerical analysts, we will now give a brief, heuristic derivation of the boundary value problem for the first-passage time $T(x, y)$ of the random function $\xi(t)$ satisfying the stochastic differential equation (1.5). This is a tutorial derivation, for which we claim neither originality nor rigor.

Let $p(x, y, t)$ be the probability-density of the time $t$ at which the particle first crosses the boundary $\xi= \pm 1$. Thus, $p \geqq 0$ and $\int_{0}^{\infty} p d t=1$. In a small time increment $\Delta t$, the particle with position $x$ and velocity $y$ moves to the position $x+y \Delta t$, while the velocity changes from $y$ to $y+g$, where $g$ is the random Gaussian variate

$$
g=\int_{t}^{t+\Delta t} w(\tau) d \tau
$$

By the definition of white noise, $g$ has mean 0 and has variance $\Delta t$. Thus, we may write $g=\sqrt{\Delta t} r$, where $r$ is a standard Gaussian variate with zero mean and unit variance.

The particle with initial position and velocity $x$ and $y$ will cross the boundary in time $t$ if and only if the particle with initial position and velocity $x+\Delta t y$ and $y+\sqrt{\Delta t} r$ will cross the boundary in time $t-\Delta t$. Averaging over the random variable $r$, we obtain the identity for small $\Delta t$ :

$$
p(x, y, t)=\int_{-\infty}^{\infty} p(x+\Delta t y, y+r \sqrt{\Delta t}, t-\Delta t) \cdot \frac{e^{-r^{2} / 2}}{\sqrt{2 \pi}} d r .
$$

Expanding the integrand, we find

$$
\begin{aligned}
p=\int_{-\infty}^{\infty}\left[p+\sqrt{\Delta t} r \frac{\partial p}{\partial y}+\Delta t\left(y \frac{\partial p}{\partial x}+\frac{1}{2} r^{2} \frac{\partial^{2} p}{\partial y^{2}}-\frac{\partial p}{\partial t}\right)\right] \frac{e^{-r^{2} / 2}}{\sqrt{2 \pi}} d r & \\
& +O\left((\Delta t)^{3 / 2}\right) .
\end{aligned}
$$

Since $r$ has integral 0 , and $r^{2}$ has integral 1, we find

$$
p=p+\Delta t\left(y \frac{\partial p}{\partial x}+\frac{1}{2} \frac{\partial^{2} p}{\partial y^{2}}-\frac{\partial p}{\partial t}\right)+O\left((\Delta t)^{3 / 2}\right) .
$$


Dividing by $\Delta t$ and letting $\Delta t \rightarrow 0$, we obtain the partial differential equation

$$
0=y \frac{\partial p}{\partial x}+\frac{1}{2} \frac{\partial^{2} p}{\partial y^{2}}-\frac{\partial p}{\partial t}
$$

for the density $p(x, y, t)$. Define the expected value

$$
T(x, y)=\int_{0}^{\infty} t p(x, y, t) d t .
$$

Multiplying (2.2) by $t$ and integrating, we find

$$
0=y \frac{\partial}{\partial x} \int_{0}^{\infty} t p d t+\frac{1}{2} \frac{\partial^{2}}{\partial y^{2}} \int_{0}^{\infty} t p d t-\int_{0}^{\infty} t \frac{\partial p}{\partial t} d t .
$$

But, for every $A>0$,

$$
-\int_{0}^{A} t \frac{\partial p}{\partial t} d t=-A p(x, y, A)+\int_{0}^{A} p(x, y, t) d t .
$$

Since $p \geqq 0$, and since $\int_{0}^{\infty} p d t=1<\infty$, we must have

$$
\liminf _{A \rightarrow \infty} A p(x, y, A)=0 .
$$

Therefore,

$$
-\int_{0}^{\infty} t \frac{\partial p}{\partial t} d t=\int_{0}^{\infty} p d t=1
$$

and the identity (2.4) yields

$$
0=y \frac{\partial T}{\partial x}+\frac{1}{2} \frac{\partial^{2} T}{\partial y^{2}}+1 .
$$

When $x=1$, we shall have $T(x, y)=0$ if $y>0$; for then the particle is at the right-hand boundary and is moving to the right. Similarly, we shall have $T(-1, y)=0$ if $y<0$. Thus we have obtained the boundary value problem $(2.2),(2.3),(2.4)$. Fichera has shown that this problem is well-posed.

To allay any last doubt about the boundary value problem as a description of the random process $\xi(t)$, we performed Monte Carlo calculations based solely on the stochastic differential equation. These calculations yielded the numerical value

$$
\bar{T}(0,0)=2.343236
$$

with an empirical standard deviation 0.0684 for $\bar{T}$. By comparison, the 
value from the analytic solution of the boundary value problem is

$$
T(0,0)=2.3120 \text {. }
$$

2.1. Similar solutions. From the solution $T(x, y)$ for the strip between $x=-1$ and $x=1$ we can construct a solution $T(x, y, a)$ for the strip between $x=-a$ and $x=a$ by the identity

$$
T(x, y, a)=a^{2 / 3} T\left(a^{-1} x, a^{-1 / 3} y\right) .
$$

The reader will readily verify that this function also satisfies the differential equation (2.5), and that this function equals zero when $x=a$ for $y>0$, and when $x=-a$ for $y<0$. In particular, we find

$$
T(0,0, a)=a^{2 / 3} \cdot T(0,0)=2.3120 a^{2 / 3}
$$

for the expected time for a randomly accelerated particle, starting from rest, to attain the distance $a$ from its starting point.

3. Relation to classical Brownian motion. In discussing Brownian motion, we consider a particle with random velocity:

$$
\frac{d \xi}{d t}=w(t), \quad t>0 .
$$

If the initial position is $x$, with $|x|<1$, and if $p(x, t)$ is the probability density of the time $t$ at which $\xi$ first equals \pm 1 , the preceding type of reasoning yields the partial differential equation

$$
0=\frac{1}{2} \frac{\partial^{2} p}{\partial x^{2}}-\frac{\partial p}{\partial t}
$$

Multiplying this equation by $t$ and integrating, we find

$$
0=\frac{1}{2} \frac{d^{2}}{d x^{2}} T(x)+1
$$

The boundary conditions are $T( \pm 1)=0$, and the solution is therefore $T(x)=1-x^{2}$; cf. [6].

4. Analytic solution. We now consider the problem of obtaining an analytic representation of the first passage time $T(x, y)$. It will be obtained as the solution of the boundary value problem

(4.1) $\frac{1}{2} \frac{\partial^{2} T}{\partial y^{2}}+y \frac{\partial T}{\partial x}+1=0, \quad-1<x<1$,

(4.2) $T(x, y)$ bounded, $\quad T(x, y)=O\left(|y|^{-1}\right)$ as $|y| \rightarrow \infty$,

$$
\begin{aligned}
& T(1, y)=0, \quad y>0, \\
& T(-1, y)=0, \quad y<0 .
\end{aligned}
$$


First we show that the boundary conditions apply to $T(x, y)$, and that the solution of (1)-(3) is unique. Then the solution will be derived.

It follows from the stochastic differential equation $\ddot{x}=w(t)$ that for initial conditions $(x, y)$, the position of the particle at time $t$ is a normal random variable with mean $x+t y$, variance $t^{3} / 3$. Thus the probability $P(x, y, t)$ that the time of escape from the interval $(-1,1)$ is greater than $t$ can be estimated in terms of the probability of being in the interval at time $t$ :

$$
P(x, y, t) \leqq \Phi\left[3^{1 / 2} t^{-3 / 2}(x+t y+1)\right]-\Phi\left[3^{1 / 2} t^{-3 / 2}(x+t y-1)\right],
$$

where $\Phi$ is the Gaussian distribution function

$$
\Phi(z)=\frac{1}{\sqrt{2 \pi}} \int_{-\infty}^{z} \exp \left(-\frac{1}{2} s^{2}\right) d s .
$$

In particular,

$$
P(x, y, t) \leqq \min \left\{1,(6 / \pi)^{1 / 2} t^{-3 / 2}\right\},
$$

which is enough to show that

$$
T(x, y)=\int_{0}^{\infty} P(x, y, t) d t
$$

is uniformly bounded: $T(x, y) \leqq 3(6 / \pi)^{1 / 3}$.

For large positive $y$, consider (4.4) at $t=(2-x) / y$. Both arguments of $\Phi$ are positive. Using the estimate

$$
\Phi\left(z_{2}\right)-\Phi\left(z_{1}\right)<\frac{C_{1} \exp \left(-z_{1}^{2} / 2\right)}{z_{1}}, \quad 0<z_{1}<z_{2}
$$

( $C_{1}$ a constant), we have

$$
\begin{aligned}
P(x, y,(2-x) / y) & <C_{1} 3^{-1 / 2}(2-x)^{3 / 2} y^{-3 / 2} \exp \left[-3(2-x)^{-3} y^{3} / 2\right] \\
& <3 C_{1} y^{-3 / 2} \exp \left(-y^{3} / 18\right) .
\end{aligned}
$$

Since $P$ is a decreasing function of $t$, we have

$$
P(x, y, t)<3 C_{1} y^{-3 / 2} \exp \left(-y^{3} / 18\right), \quad t \geqq(2-x) / y .
$$

Using this together with (4.5), we find for $y>3$,

$$
\begin{aligned}
T(x, y)< & \int_{0}^{(2-x) / y} 1 \cdot d t+\int_{(2-x) / y}^{\exp \left(y^{3} / 27\right)} 3 C_{1} y^{-3 / 2} \exp \left(-\frac{1}{18} y^{3}\right) d t \\
& +\int_{\exp \left(y^{3} / 27\right)}^{\infty}\left(\frac{6}{\pi}\right)^{1 / 2} t^{-3 / 2} d t \\
= & (2-x) / y+O\left[\exp \left(-y^{3} / 54\right)\right] \text { as } y \rightarrow \infty .
\end{aligned}
$$


A similar procedure can be used to show that $T(x, y) \rightarrow 0$ as $(x, y) \rightarrow\left(1, y_{0}\right), y_{0}>0$. By symmetry, the conditions of (4.2) and (4.3) for negative $y$ are also valid.

For uniqueness, we appeal to a theorem of Fichera [1]. Let two solutions of (4.1)-(4.3) differ by $u(x, y)$. Then

$$
\frac{1}{2} \frac{\partial^{2} u}{\partial y^{2}}+y \frac{\partial u}{\partial x}=0
$$

and $u$ satisfies (4.2) and (4.3). We apply Fichera's Theorem XI [1, p. 108] to $u$ in the region

$$
-a<x<a, \quad-b<y<b,
$$

where $0<a<1,0<b<\infty$. In the notation of Fichera, we take $p=2$, $w(x, y)=y^{2}-b^{2}$. After letting $a \rightarrow 1$ in the result of this theorem, we have the inequality

$$
\iint_{|x|<1,|y|<b} u^{2} d x d y \leqq b \int_{-1}^{1}\left[u(x, b)^{2}+u(x,-b)^{2}\right] d x
$$

which is $O\left(b^{-1}\right)$ since $u$ satisfies (4.2). Letting $b \rightarrow \infty$, we conclude that $u$ is identically zero.

Now that uniqueness is known, we can make any plausible assumptions along the way in solving (4.1)-(4.3). These will be justified if we finally get a solution.

We notice first that $T(x, y)=T(-x,-y)$, and this relation can be used to generate $T$ in the whole strip $-1<x<1$ from its values in the half-strip $y \geqq 0,-1<x<1$. For a boundary value problem for $T(x, y)$ in the upper half-strip, we can take (4.1) and the conditions of (4.2) and (4.3) for $y>0$, along with

$$
\begin{aligned}
T(x, 0) & =T(-x, 0), \\
T_{y}(x, 0) & =-T_{y}(-x, 0)
\end{aligned}
$$

for conditions on the $x$-axis. Then when $T(x, y)$ is extended to the whole strip by symmetry, it will satisfy (4.1)-(4.3).

We express $T(x, y)$ for $y \geqq 0$ as the sum of two functions,

$$
T(x, y)=u(x, y)+v(x, y)
$$

where $u(x, y)$ is the solution of (4.1) with the boundary conditions

$$
\begin{aligned}
& u(x, y) \text { bounded, } \quad u(x, y)=O\left(y^{-1}\right) \text { as } y \rightarrow \infty, \\
& u(1, y)=0, \quad y>0,
\end{aligned}
$$


and

$$
u(x, 0)=0, \quad-1<x<1 .
$$

Then $v(x, y)$ satisfies the homogeneous equation

$$
\frac{1}{2} \frac{\partial^{2} v}{\partial y^{2}}+y \frac{\partial v}{\partial x}=0
$$

It satisfies the boundary conditions (4.9), and to make $T$ satisfy (4.7), we need

$$
\begin{aligned}
& v(x, 0)=v(-x, 0)(=T(x, 0)), \\
& v_{y}(x, 0)+v_{y}(-x, 0)=-u_{y}(x, 0)-u_{y}(-x, 0) .
\end{aligned}
$$

First we shall find $u(x, y)$.

Note that if $u(x, y)$ is known, the function

$$
\lambda^{-2} u\left(1-\lambda^{3}(1-x), \lambda y\right),
$$

for $0<\lambda<1$, is also a solution of the boundary value problem for $u(x, y)$. If we identify this function with $u(x, y)$, then

$$
u(x, y)=(1-x)^{2 / 3} f(z),
$$

where

$$
z=y^{3} /(1-x) .
$$

Inserting (4.13) into the differential equation (4.1), we get an ordinary differential equation for $f(z)$ :

$$
\frac{9}{2} z f^{\prime \prime}+(3+z) f^{\prime}-\frac{2}{3} f=-z^{-1 / 3} .
$$

A particular solution of this equation is $f=-z^{2 / 3}$, corresponding to the solution $-y^{2}$ of (4.1). The associated homogeneous equation is a confluent hypergeometric equation, whose general solution can be written down. Thus we can get the general solution of (4.14). The boundary conditions (4.9) and (4.10) imply that

$$
f(0)=0=f(\infty),
$$

which makes the solution unique. All the pertinent formulas are in [3, $\S 13.1]$. We get

$$
u(x, y)=-y^{2}+A_{1}(1-x)^{2 / 3} y M\left(-\frac{1}{3}, \frac{4}{3},-\frac{2}{9} \frac{y^{3}}{1-x}\right),
$$

where

$$
A_{1}=6^{2 / 3} \Gamma(2 / 3) / \Gamma(1 / 3)
$$


and $M$ is the Kummer confluent hypergeometric function. Using the asymptotic expansion of $M$ [3, Equation 13.5.1], we find that

$$
u(x, y)=\frac{1-x}{y}+o\left(\frac{(1-x)^{2}}{y^{4}}\right)
$$

as $x \rightarrow 1$ or $y \rightarrow \infty$.

To express $v(x, y)$ in terms of its unknown boundary values $v(x, 0)$ $=v(x)$, we need a formula for the solution of (4.11) in the upper halfplane with arbitrary values on the $x$-axis. This is given by

$$
v(x, y)=\int_{-\infty}^{\infty} g(x-\xi, y) v(\xi, 0) d \xi,
$$

if $g(x, y)$ is the solution of (4.11) which approaches $\delta(x)$ as $y \rightarrow 0$.

Observe that $\lambda^{3} g\left(\lambda^{3} x, \lambda y\right), 0<\lambda<\infty$, also has the properties needed for $g(x, y)$. Assuming that these functions are the same, we get

$$
g(x, y)=-x^{-1} h(p)
$$

where

$$
p=-y^{3} / x
$$

Inserting (4.19) in (4.11), we get

$$
\frac{9}{2} p h^{\prime \prime}+(3+p) h^{\prime}+h=0 .
$$

This is again a confluent hypergeometric equation. Its general solution $[3, \S 13.1]$ is

$$
h(p)=A_{2} p^{1 / 3} \exp \left(-\frac{2}{9} p\right)+A_{3} M\left(1, \frac{2}{3},-\frac{2}{9} p\right) .
$$

On the line $x=0, p=\infty$. We need to determine $h(p)$ separately in each quadrant and to piece these functions together to get $g(x, y)$. The following conditions are certainly necessary:

$$
h(0 \pm)=0, \quad h(p) \rightarrow 0 \quad \text { as } \quad p \rightarrow \pm \infty .
$$

These conditions make all the constants but one zero, and we get

$$
g(x, y)= \begin{cases}\frac{A_{2} y}{(-x)^{4 / 3}} \exp \left(\frac{2}{9} \frac{y^{3}}{x}\right), & x<0, \\ 0, & x \geqq 0 .\end{cases}
$$

This function is $C^{\infty}$ in the upper half-plane. By an obvious change of variable,

$$
\int_{-\infty}^{\infty} g(x, y) d x=\left(\frac{9}{2}\right)^{1 / 3} A_{2} \int_{0}^{\infty} t^{-2 / 3} e^{-t} d t=6^{2 / 3} A_{2} \frac{\Gamma(1 / 3)}{2} .
$$


Thus $g(x, y) \rightarrow \delta(x)$ as $y \rightarrow 0$ if

$$
A_{2}=\frac{2}{6^{2 / 3} \Gamma(1 / 3)} .
$$

Notice that $v(x, y)$ as given by (4.18) is automatically zero on the line $x=1$ if $v(\xi, 0)=0$ for $\xi>1$. Hence

$$
v(x, y)=\int_{x}^{1} g(x-\xi, y) v(\xi) d \xi
$$

is the solution we are seeking for (4.11) if it is bounded and $O\left(y^{-1}\right)$ as $y \rightarrow \infty$. These conditions are both satisfied if $v(\xi)$ is bounded, from the formula (4.20). In fact,

as $y \rightarrow \infty$, and

$$
\begin{aligned}
|v(x, y)| & \leqq \sup |v(\xi)| \int_{x}^{1} g(x-\xi, y) d \xi \\
& =O\left[(1-x)^{2 / 3} y^{-1} \exp \left(-\frac{2}{9} \frac{y^{3}}{1-x}\right)\right]
\end{aligned}
$$

$$
|v(x, y)| \leqq \sup |v(\xi)|
$$

throughout the half-strip.

For $x<0$ we have

Hence

$$
\frac{\partial}{\partial y} g(x, y)=\frac{\partial}{\partial x}\left[3 A_{2}(-x)^{-1 / 3} \exp \left(\frac{2}{9} \frac{y^{3}}{x}\right)\right] .
$$

$$
v_{y}(x, y)=-3 A_{2} \int_{x}^{1} \frac{\partial}{\partial \xi}\left[(\xi-x)^{-1 / 3} \exp \left(-\frac{2}{9} \frac{y^{3}}{\xi-x}\right)\right] v(\xi) d \xi .
$$

We assume that $v(\xi) \rightarrow 0$ as $\xi \rightarrow 1$, an assumption which is plausible from the probabilistic interpretation of $T(x, y)$. This will be justified in the end by the uniqueness of the solution of (4.1)-(4.3). Then, by integration by parts followed by $y \rightarrow 0$, we get

$$
v_{y}(x, 0)=3 A_{2} \int_{x}^{1}(\xi-x)^{-1 / 3} v^{\prime}(\xi) d \xi .
$$

The unknown function $v(x)$ is supposed to be even and bounded, with $v( \pm 1)=0$. The only remaining condition to be satisfied is the last equation of (4.12). Transforming the integral for $v_{y}(-x, 0)$ by the symmetry of $v$, and using (4.15), we see that this equation becomes

$$
\begin{aligned}
\int_{-1}^{x} v^{\prime}(\xi)(x-\xi)^{-1 / 3} d \xi-\int_{x}^{1} v^{\prime}(\xi) & (\xi-x)^{-1 / 3} d \xi \\
= & \frac{A_{1}}{3 A_{2}}\left[(1-x)^{1 / 3}+(1+x)^{1 / 3}\right]
\end{aligned}
$$


The integral equation (4.24) is a special case of an equation considered by Sakalyuk [4]. ${ }^{1}$ The method of solution given in [4] proceeds as follows: Let our equation be

$$
\begin{aligned}
\int_{-1}^{x} f(\xi)(x-\xi)^{-1 / 3} d \xi-\int_{x}^{1} f(\xi)(\xi-x)^{-1 / 3} d \xi= & g(x) \\
& -1<x<1 .
\end{aligned}
$$

We consider the function

$$
F(z)=\left(z^{2}-1\right)^{1 / 6} \int_{-1}^{1} \frac{f(\xi) d \xi}{(z-\xi)^{1 / 3}}
$$

for complex $z \notin[-1,1]$, where the roots are both asymptotic to the same branch of $z^{1 / 3}$ at infinity. For $-1<x<1$, let

$$
\begin{aligned}
& F_{+}(x)=\lim _{y \rightarrow 0+} F(x+i y), \\
& F_{-}(x)=\lim _{y \rightarrow 0-} F(x+i y) .
\end{aligned}
$$

These limits exist if we suppose, for example, that $f(x)$ is locally bounded on $(-1,1)$. Then

$$
\begin{aligned}
F_{+}(x)=\left(1-x^{2}\right)^{1 / 6} & \\
\cdot & \left\{e^{\pi i / 6} \int_{-1}^{x} f(\xi)(x-\xi)^{-1 / 3} d \xi+e^{-\pi i / 6} \int_{x}^{1} f(\xi)(\xi-x)^{-1 / 3} d \xi\right\},
\end{aligned}
$$

and $F_{-}(x)$ is given by the complex conjugate expression. Two useful combinations of these functions are

$$
F_{+}(x)-F_{-}(x)=i\left(1-x^{2}\right)^{1 / 6} g(x)
$$

(from (4.25)) and

$$
\begin{aligned}
\int_{-1}^{x} f(\xi)(x & -\xi)^{-1 / 3} d \xi \\
& =\frac{1}{i \sqrt{3}}\left(1-x^{2}\right)^{-1 / 6}\left[e^{\pi i / 6} F_{+}(x)-e^{-\pi i / 6} F_{-}(x)\right] .
\end{aligned}
$$

Equation (4.27) is satisfied by the function

$$
F^{*}(z)=\frac{1}{2 \pi} \int_{-1}^{1} \frac{d \xi}{\xi-z}\left(1-\xi^{2}\right)^{1 / 6} g(\xi),
$$

if $g(\xi)$ is differentiable for $-1<\xi<1$. The function $F(z)-F^{*}(z)$ is

1 Fleming [2] uses a different method of solution which leads to a different (but also correct) integral formula. 
easily shown to be entire and bounded; hence it is a constant. In the application to (4.24), $f(x)=v^{\prime}(x)$, an odd function. Then $F$ and $F^{*}$ both apbroach zero as $z \rightarrow \infty$; hence $F=F^{*}$. Using this function in (4.28), we have

$$
\begin{aligned}
\int_{-1}^{x} f(\xi)( & x-\xi)^{-1 / 3} d \xi \\
= & \frac{1}{2} g(x)+\frac{1}{2 \pi \sqrt{3}}\left(1-x^{2}\right)^{-1 / 6} \int_{-1}^{1} \frac{d \xi}{\xi-x}\left(1-\xi^{2}\right)^{1 / 6} g(\xi),
\end{aligned}
$$

where the Cauchy principal value of the integral at $\xi=x$ is understood. Thus the solution of (4.25) is reduced to the solution of an Abel equation.

We now apply this result to (4.24). We have

$$
\int_{-1}^{x} v^{\prime}(\xi)(x-\xi)^{-1 / 3} d \xi=\frac{A_{1}}{6 A_{2}} k(x),
$$

where

$$
\begin{aligned}
& k(x)=(1-x)^{1 / 3}+(1+x)^{1 / 3} \\
& +\frac{1}{\pi \sqrt{3}}\left(1-x^{2}\right)^{-1 / 6} \int_{-1}^{1} \frac{d \xi}{\xi-x}\left(1-\xi^{2}\right)^{1 / 6}\left[(1-\xi)^{1 / 3}+(1+\xi)^{1 / 3}\right] .
\end{aligned}
$$

By integrating along a line in the complex plane just above the real axis, we can deduce that

$$
\int_{-1}^{1} \frac{d \xi(1+\xi)^{1 / 6}}{(\xi-x)(1-\xi)^{1 / 2}}=-\frac{\sqrt{3}}{2} \int_{-\infty}^{-1} \frac{d \xi(-\xi-1)^{1 / 6}}{(\xi-x)(-\xi+1)^{1 / 2}} .
$$

Putting $\xi=1-2 / t$ in this integral reduces it to the standard integral for the hypergeometric function [3, Equation 15.3.1]. We get

$$
\int_{-1}^{1} \frac{d \xi(1+\xi)^{1 / 6}}{(\xi-x)(1-\xi)^{1 / 2}}=2^{-4 / 3} 3^{1 / 2} B\left(\frac{1}{3}, \frac{7}{6}\right) F\left(1, \frac{1}{3}, \frac{3}{2}, \frac{1-x}{2}\right),
$$

where $B$ is the beta function. Hence

$$
\begin{aligned}
\int_{-1}^{1} \frac{d \xi}{\xi-x} & \left(1-\xi^{2}\right)^{1 / 6}(1-\xi)^{1 / 3} \\
= & (1-x) \int_{-1}^{1} \frac{d \xi(1+\xi)^{1 / 6}}{(\xi-x)(1-\xi)^{1 / 2}}-\int_{-1}^{1} d \xi \frac{(1+\xi)^{1 / 6}}{(1-\xi)^{1 / 2}} \\
& =2^{-4 / 3} 3^{1 / 2} B\left(\frac{1}{3}, \frac{7}{6}\right)(1-x) F\left(1, \frac{1}{3}, \frac{3}{2}, \frac{1-x}{2}\right)-2^{2 / 3} B\left(\frac{1}{2}, \frac{7}{6}\right) .
\end{aligned}
$$

The other integral needed in (4.31) has a similar formula, obtained from 
this by changing the signs of $x$ and $\xi$. Thus

$$
\begin{array}{r}
k(x)=2^{-4 / 3} \pi^{-1} B\left(\frac{1}{3}, \frac{7}{6}\right)\left(1-x^{2}\right)^{-1 / 6} \\
\cdot\left[(1-x) F\left(1, \frac{1}{3}, \frac{3}{2}, \frac{1-x}{2}\right)-(1+x) F\left(1, \frac{1}{3}, \frac{3}{2}, \frac{1+x}{2}\right)\right] \\
+(1-x)^{1 / 3}+(1+x)^{1 / 3} .
\end{array}
$$

To find $v(x)$, multiply $(4.30)$ by $(z-x)^{-2 / 3}$ and integrate over $x$ from -1 to $z$. On the left, we have

$$
\int_{-1}^{z} d \xi v^{\prime}(\xi) \int_{\xi}^{z} \frac{d x}{(z-x)^{2 / 3}(x-\xi)^{1 / 3}}=\frac{2 \pi}{\sqrt{3}} v(z)
$$

Hence

$$
v(x)=\frac{A_{1}}{4 \pi \sqrt{3} A_{2}} \int_{-1}^{x}(x-\xi)^{-2 / 3} k(\xi) d \xi .
$$

To evaluate this integral, different devices are required for the various terms in (4.32). First we consider

$$
\begin{array}{r}
I_{1}(x)=\int_{-1}^{x} d \xi(x-\xi)^{-2 / 3}(1-\xi)^{-1 / 6}(1+\xi)^{5 / 6} \\
\cdot F\left(1, \frac{1}{3}, \frac{3}{2}, \frac{1+\xi}{2}\right) .
\end{array}
$$

By [3, Equation 15.3.3],

$$
F\left(1, \frac{1}{3}, \frac{3}{2}, \frac{1+\xi}{2}\right)=\left(\frac{1-\xi}{2}\right)^{1 / 6} F\left(\frac{1}{2}, \frac{7}{6}, \frac{3}{2}, \frac{1+\xi}{2}\right) .
$$

Hence, after the substitution $\xi=-1+t(1+x)$, we have

$$
I_{1}(x)=2^{-1 / 6}(1+x)^{7 / 6} \int_{0}^{1} d t(1-t)^{-2 / 3} t^{5 / 6} F\left(\frac{1}{2}, \frac{7}{6}, \frac{3}{2}, \frac{t(1+x)}{2}\right) .
$$

Using the power series expansion

$$
F\left(\frac{1}{2}, \frac{7}{6}, \frac{3}{2}, z\right)=\frac{1}{2} \sum_{n=0}^{\infty} \frac{\Gamma(n+7 / 6)}{n ! \Gamma(1 / 6)} \frac{z^{n}}{n+1 / 2},
$$

the integration can be carried out term by term in terms of beta functions. After some reduction,

$$
I_{1}(x)=\frac{5}{8} 2^{-1 / 6}(1+x)^{7 / 6} B\left(\frac{1}{3}, \frac{5}{6}\right)
$$




$$
\begin{aligned}
\cdot \sum_{n=0}^{\infty} \frac{\Gamma(n+11 / 6)}{n ! \Gamma(11 / 6)}\left(\frac{1}{n+1 / 2}-\frac{1}{n+11 / 6}\right)\left(\frac{1+x}{2}\right)^{n} \\
=\frac{5}{4} 2^{-1 / 6} B\left(\frac{1}{3}, \frac{5}{6}\right)(1+x)^{7 / 6} \\
\cdot\left[F\left(\frac{11}{6}, \frac{1}{2}, \frac{3}{2}, \frac{1+x}{2}\right)-\frac{3}{7} F\left(\frac{11}{6}, \frac{7}{6}, \frac{13}{6}, \frac{1+x}{2}\right)\right] .
\end{aligned}
$$

These hypergeometric functions will be transformed for later combination of $I_{1}(x)$ with the other integrals. By the use of equations 15.3.3, 15.3.6, and 15.2.20 of [3], it can be shown that

$$
\begin{aligned}
z F\left(\frac{11}{6}, \frac{1}{2}, \frac{3}{2}, z\right)= & \frac{3}{5}(1-z)^{-5 / 6}+\frac{1}{5} B\left(\frac{1}{2}, \frac{1}{6}\right) z^{1 / 2} \\
& -\frac{9}{5}(1-z)^{1 / 6} F\left(1,-\frac{1}{3}, \frac{7}{6}, 1-z\right), \\
z F\left(\frac{11}{6}, \frac{7}{6}, \frac{13}{6}, z\right)= & \frac{7}{5}(1-z)^{-5 / 6}-\frac{7}{5}(1-z)^{1 / 6} F\left(\frac{1}{3}, 1, \frac{7}{6}, z\right) .
\end{aligned}
$$

Thus (4.35) can be rewritten as

$$
\begin{aligned}
& I_{1}(x)=3 \cdot 2^{-4 / 3} B\left(\frac{1}{3}, \frac{5}{6}\right)\left(1-x^{2}\right)^{1 / 6} \\
& \cdot\left[F\left(\frac{1}{3}, 1, \frac{7}{6}, \frac{1+x}{2}\right)-3 F\left(-\frac{1}{3}, 1, \frac{7}{6}, \frac{1-x}{2}\right)\right] \\
&+3 \cdot 2^{-1 / 3} \pi(1+x)^{2 / 3} .
\end{aligned}
$$

Now consider

(4.37) $\quad I_{2}(x)=\int_{-1}^{x} d \xi(x-\xi)^{-2 / 3}(1+\xi)^{-1 / 6}(1-\xi)^{5 / 6} F\left(1, \frac{1}{3}, \frac{3}{2}, \frac{1-\xi}{2}\right)$.

Replacing $\xi$ by $-\xi$ in this integral, we see that the integrand becomes the same as that of $I_{1}(-x)$. Hence

$$
\begin{aligned}
I_{2}(x) & +I_{1}(-x) \\
& =\int_{-1}^{1} d \xi(x+\xi)^{-2 / 3}(1-\xi)^{-1 / 6}(1+\xi)^{5 / 6} F\left(1, \frac{1}{3}, \frac{3}{2}, \frac{1+\xi}{2}\right) .
\end{aligned}
$$

Use here the integral formula [3, Equation 15.3.1]

$$
F\left(1, \frac{1}{3}, \frac{3}{2}, \frac{1+\xi}{2}\right)=B\left(\frac{1}{3}, \frac{7}{6}\right)^{-1} \int_{0}^{1} d z \frac{z^{-2 / 3}(1-z)^{1 / 6}}{1-z(1+\xi) / 2},
$$

and reverse the order of integration. The inner integral over $\xi$ is propor- 
tional to a contour integral in the complex plane along a closed curve around the segment $-1 \leqq \xi \leqq 1$, and can be evaluated in terms of the residues of the integrand outside the curve. We obtain

$$
\begin{aligned}
\int_{-1}^{1} \frac{d \xi(1+\xi)^{5 / 6}}{(x+\xi)^{2 / 3}(1-\xi)^{1 / 6}[1-} & z(1+\xi) / 2] \\
= & \frac{4 \pi}{z}\left\{(1-z)^{-1 / 6}\left[1-\frac{1}{2} z(1-x)\right]^{-2 / 3}-1\right\},
\end{aligned}
$$

and (4.38) becomes

$$
\begin{aligned}
& I_{2}(x)=-I_{1}(-x) \\
& +4 \pi B\left(\frac{1}{3}, \frac{7}{6}\right)^{-1} \int_{0}^{1} d z z^{-5 / 3}\left\{\left[1-\frac{1}{2} z(1-x)\right]^{-2 / 3}-(1-z)^{1 / 6}\right\} .
\end{aligned}
$$

For $b>0$, equation 15.3 .1 of [3] yields

$$
\begin{aligned}
\int_{0}^{1} d z z^{b-1}\left\{(1-x z)^{-2 / 3}\right. & \left.-(1-z)^{1 / 6}\right\} \\
= & B(b, 1) F\left(\frac{2}{3}, b, b+1, x\right)-B\left(b, \frac{7}{6}\right) .
\end{aligned}
$$

All the terms in this equation are analytic in $b$ for $\operatorname{Re} b>-1$, except at $b=0$. Hence the equation is also valid for $-1<b<0$. In (4.39), we have this integral for $b=-\frac{2}{3}$. Hence, after simplification,

$$
I_{2}(x)=-I_{1}(-x)-6 \pi B\left(\frac{1}{3}, \frac{7}{6}\right)^{-1} F^{\prime}\left(\frac{2}{3},-\frac{2}{3}, \frac{1}{3}, \frac{1-x}{2}\right)+3 \pi .
$$

The contributions to (4.33) of the other parts of $k(\xi)$ are relatively simple. Using the substitution $\xi=-1+t(1+x)$ to bring the integrals into standard forms, we have

$$
I_{3}(x)=\int_{-1}^{x} d \xi(x-\xi)^{-2 / 3}(1+\xi)^{1 / 3}=B\left(\frac{1}{3}, \frac{4}{3}\right)(1+x)^{2 / 3}
$$

and

$$
\begin{aligned}
I_{4}(x) & =\int_{-1}^{x} d \xi(x-\xi)^{-2 / 3}(1-\xi)^{1 / 3} \\
& =3 \cdot 2^{1 / 3}(1+x)^{1 / 3} F\left(-\frac{1}{3}, 1, \frac{4}{3}, \frac{1+x}{2}\right) .
\end{aligned}
$$

We find, by an application of equations 15.3 .6 and 15.3.3 of [3], that

$$
I_{4}(x)=3 \cdot 2^{-1 / 3} F\left(\frac{2}{3},-\frac{2}{3}, \frac{1}{3}, \frac{1-x}{2}\right)+B\left(\frac{1}{3}, \frac{4}{3}\right)(1-x)^{2 / 3} .
$$


We have

$$
\begin{aligned}
& \int_{-1}^{x} d \xi(x-\xi)^{-2 / 3} k(\xi) \\
&=2^{-4 / 3} \pi^{-1} B\left(\frac{1}{3}, \frac{7}{6}\right)\left[I_{2}(x)-I_{1}(x)\right]+I_{3}(x)+I_{4}(x) \\
&=2^{-4 / 3} \pi^{-1} B\left(\frac{1}{3}, \frac{7}{6}\right)\left[3 \pi-I_{1}(x)-I_{1}(-x)\right] \\
&+B\left(\frac{1}{3}, \frac{4}{3}\right)\left[(1-x)^{2 / 3}+(1+x)^{2 / 3}\right],
\end{aligned}
$$

by (4.39), (4.41) and (4.42). When $I_{1}(x)$ is eliminated by (4.36), there is some cancellation of terms. By equation 15.3.6 of [3],

$$
F\left(\frac{1}{3}, 1, \frac{7}{6}, \frac{1+x}{2}\right)+F\left(\frac{1}{3}, 1, \frac{7}{6}, \frac{1-x}{2}\right)=\frac{2^{1 / 3}}{6} B\left(\frac{1}{6}, \frac{1}{6}\right)\left(1-x^{2}\right)^{-1 / 6},
$$

and simplifying the combinations of beta functions which occur, we get

$$
\begin{aligned}
\int_{-1}^{x}(x-\xi)^{-2 / 3} k(\xi) d \xi=3^{3 / 2} 2^{-1 / 3}\left(1-x^{2}\right)^{1 / 6} & {\left[F\left(-\frac{1}{3}, 1, \frac{7}{6}, \frac{1+x}{2}\right)\right.} \\
& \left.+F\left(-\frac{1}{3}, 1, \frac{7}{6}, \frac{1-x}{2}\right)\right] .
\end{aligned}
$$

Hence from (4.33),

$$
\begin{aligned}
v(x)=C\left(1-x^{2}\right)^{1 / 6}\left[F \left(-\frac{1}{3}, 1, \frac{7}{6},\right.\right. & \left.\frac{1+x}{2}\right) \\
& \left.+F\left(-\frac{1}{3}, 1, \frac{7}{6}, \frac{1-x}{2}\right)\right],
\end{aligned}
$$

where

$$
C=\frac{3^{3 / 2} 2^{-1 / 3} A_{1}}{4 \pi \sqrt{3} A_{2}}=\frac{3^{11 / 6}}{2 \Gamma(1 / 3)},
$$

by (4.16) and (4.21).

Collecting the formulas for $T(x, y)$ in the upper half-strip, we have

$$
T(x, 0)=v(x)
$$

given in (4.43), and for $y>0$,

$$
\begin{aligned}
T(x, y)= & -y^{2}+A_{1}(1-x)^{1 / 3} y M\left(-\frac{1}{3}, \frac{4}{3},-\frac{2}{9} \frac{y^{3}}{1-x}\right) \\
& +A_{2} y \int_{x}^{1} \frac{d \xi}{(\xi-x)^{4 / 3}} \exp \left(-\frac{2}{9} \frac{y^{3}}{\xi-x}\right) T(\xi, 0),
\end{aligned}
$$

where $A_{1}$ and $A_{2}$ are given in (4.16) and (4.21). 
The hypergeometric function in (4.43) has a negative second derivative. Thus $T(x, 0)$ has the form

$$
T(x, 0)=C\left(1-x^{2}\right)^{1 / 6} m(x),
$$

where $m(x)$ is a concave, even function, which has a positive limiting value at $x= \pm 1$. This shows that $T(x, y)$ cannot have bounded partial derivatives near $( \pm 1,0)$. To investigate the behavior of $T(x, y)$ near $(1,0)$ more closely, we first transform the hypergeometric function with argument $(1+x) / 2$ in (4.43), according to formula 15.3.6 of [3]:

$$
\begin{aligned}
F\left(-\frac{1}{3}, 1, \frac{7}{6}, \frac{1+x}{2}\right)= & \frac{1}{3} F\left(-\frac{1}{3}, 1, \frac{1}{2}, \frac{1-x}{2}\right) \\
& +\frac{2^{-1 / 3}}{9} B\left(\frac{1}{6}, \frac{1}{2}\right)(1-x)^{1 / 2}(1+x)^{-1 / 6} .
\end{aligned}
$$

Then we have

$$
\begin{aligned}
T(x, 0) & =C\left(1-x^{2}\right)^{1 / 6}\left[F\left(-\frac{1}{3}, 1, \frac{7}{6}, \frac{1-x}{2}\right)\right. \\
+ & \left.\frac{1}{3} F\left(-\frac{1}{3}, 1, \frac{1}{2}, \frac{1-x}{2}\right)\right]+\frac{2^{-1 / 3}}{9} C B\left(\frac{1}{6}, \frac{1}{2}\right)(1-x)^{2 / 3},
\end{aligned}
$$

which can be expanded in a series of increasing powers of $(1-x)$, convergent for $x>-1$. If we insert this series in the last term $v(x, y)$ in (4.45), and integrate term by term, we obtain a series expansion of $v(x, y)$ which converges in the interior of the upper half-strip, and which is dominated by the series for $T(x, 0)$.

The first terms in the expansion of (4.46) give us

Hence

$$
T(x, 0)=\frac{4}{3} 2^{1 / 6} C(1-x)^{1 / 6}+O\left((1-x)^{2 / 3}\right) .
$$

where

$$
\begin{aligned}
& v(x, y)= {\left[1+O\left((1-x)^{1 / 2}\right)\right] A_{3} y } \\
& \cdot \int_{x}^{1} \frac{d \xi}{(\xi-x)^{4 / 3}} \exp \left(-\frac{2}{9} \frac{y^{3}}{\xi-x}\right)(1-\xi)^{1 / 6},
\end{aligned}
$$

$$
A_{3}=\frac{4}{3} 2^{1 / 6} C A_{2} .
$$

This integral can be expressed in terms of the confluent hypergeometric function of the second kind, $U(a, b, z)$ (see [3, Formula 13.1.3]). Making the substitution

$$
\xi=x+\frac{1-x}{t},
$$


we can apply equation 13.2.6 of [3] to get

$$
\begin{gathered}
v(x, y)=A_{3} \Gamma\left(\frac{7}{6}\right) y(1-x)^{-1 / 6} e^{-z} U\left(\frac{7}{6}, \frac{4}{3}, z\right) \\
\cdot\left[1+O\left((1-x)^{1 / 2}\right)\right],
\end{gathered}
$$

where

$$
z=\frac{2}{9} \frac{y^{3}}{1-x} .
$$

This can be expressed in terms of the function $M(a, b, z)$ by equations 13.1.3 and 13.1.27 of [3] as

$$
\begin{aligned}
v(x, y)= & 2 \pi \sqrt{3} A_{3}\left\{\frac{6^{-1 / 3}}{\Gamma(2 / 3)}(1-x)^{1 / 6} M\left(-\frac{1}{6}, \frac{2}{3},-z\right)\right. \\
- & \left.B\left(\frac{1}{3}, \frac{5}{6}\right)^{-1} y(1-x)^{-1 / 6} M\left(\frac{1}{6}, \frac{4}{3},-z\right)\right\} \\
& \cdot\left[1+O\left((1-x)^{1 / 2}\right)\right] .
\end{aligned}
$$

A formula similar to $(4.47)$ holds near $(-1,0)$. It can be shown that as $(x, y) \rightarrow(-1,0)$,

(4.50) $\quad v(x, y) \sim A_{3} y \int_{x}^{1} \frac{d \xi}{(\xi-x)^{4 / 3}} \exp \left(-\frac{2}{9} \frac{y^{3}}{\xi-x}\right)(1+\xi)^{1 / 6}$.

This integral is asymptotically the same if the upper limit is replaced by infinity, which gives another expression involving confluent hypergeometric functions. Using the symmetry of $T(x, y)$, we find that $T(x, y)$ has the same asymptotic form as given in $(4.49)$ when $(x, y) \rightarrow(1,0)$ with $y<0$.

It can be shown that the dominant terms in the derivatives of $T(x, y)$ near $(1,0)$ are given by the derivatives of the expression in (4.49) and the first terms in (4.45) (these enter only for $y>0$ ). These derivatives are so poorly behaved that the truncation error for any of the difference methods discussed is unbounded as $\Delta x, \Delta y \rightarrow 0$. In fact, if we take $\Delta x=\Delta y=h$, the $L_{p}$-norm of the truncation error for any $p \geqq 1$ becomes infinite as $h \rightarrow 0$.

Equation (4.50) gives the asymptotic form of $T(x, y)$ as $(x, y) \rightarrow(-1,0)$ with $y>0$. In particular, this is true on the line $x=-1$, where we have

$$
\begin{aligned}
T(-1, y) & \sim A_{3} y \int_{-1}^{\infty} d \xi(1+\xi)^{-7 / 6} \exp \left(-\frac{2}{9} \frac{y^{3}}{1+\xi}\right) \\
& =A_{3} \Gamma\left(\frac{1}{6}\right)\left(\frac{9}{2}\right)^{1 / 6} y^{1 / 2} .
\end{aligned}
$$

For the behavior of $T(x, y)$ as $y \rightarrow \infty$, note that $v(x, y)$ decreases ex- 
ponentially. Thus the asymptotic form of $T$ is given by (4.16), and

$$
T(x, y)=\frac{1-x}{y}+O\left(y^{-4}\right) .
$$

5. The Monte Carlo solution. To obtain a value for $T(0,0)$ directly from the stochastic differential equation $\xi^{\prime \prime}(t)=w(t)$, we note that

$$
\begin{aligned}
& \xi(t+\Delta t)=\xi(t)+\Delta t \xi^{\prime}(t)+\int_{t}^{t+\Delta t}(t+\Delta t-\tau) w(\tau) d \tau, \\
& \xi^{\prime}(t+\Delta t)=\xi^{\prime}(t)+\int_{t}^{t+\Delta t} w(\tau) d \tau .
\end{aligned}
$$

Since $E w(\tau) w(s)=\delta(\tau-s)$, we find

$$
\begin{aligned}
\Delta \xi & =\xi^{\prime} \cdot \Delta t+g_{1}, \\
\Delta \xi^{\prime} & =g_{2},
\end{aligned}
$$

where $g_{1}$ and $g_{2}$ are Gaussian variates with means zero and with covariance matrix

$$
E\left[\begin{array}{l}
g_{1} \\
g_{2}
\end{array}\right]\left[\begin{array}{ll}
g_{1} & g_{2}
\end{array}\right]=\left[\begin{array}{ll}
\frac{1}{3}(\Delta t)^{3} & \frac{1}{2}(\Delta t)^{2} \\
\frac{1}{2}(\Delta t)^{2} & \Delta t
\end{array}\right] .
$$

Thus, we may set

$$
\begin{aligned}
& g_{1}=\frac{1}{\sqrt{3}}(\Delta t)^{3 / 2} z_{1}, \\
& g_{2}=\frac{1}{2} \sqrt{3 \Delta t} z_{1}+\frac{1}{2} \sqrt{\Delta t} z_{2}
\end{aligned}
$$

if $z_{1}$ and $z_{2}$ are uncorrelated Gaussian variates with zero means and unit variances. Numerical samples of $z_{1}$ and $z_{2}$ can be generated by the technique of Box and Muller [10].

We can estimate $T(0,0)$ by the sample mean

$$
\bar{T}=\frac{1}{M}\left(T_{1}+T_{2}+\cdots+T_{M}\right),
$$

where $M$ is some large integer, and where each $T_{i}$ is computed independently as follows: An increment $\Delta t$ is picked. The numbers $\xi$ and $\xi^{\prime}$ are set initially equal to zero. We let

$$
\begin{aligned}
\xi((n+1) \Delta t) & =\xi(n \Delta t)+\Delta \xi \\
\xi^{\prime}((n+1) \Delta t) & =\xi^{\prime}(n \Delta t)+\Delta \xi^{\prime}
\end{aligned}
$$


where $\Delta \xi$ and $\Delta \xi^{\prime}$ are defined by (5.1) and (5.2). Let $N_{i}$ be the first integer for which $\left|\xi\left(N_{i} \Delta t\right)\right| \geqq 1$. Since the first crossing took place between the times $\left(N_{i}-1\right) \Delta t$ and $N_{i} \Delta t$, we make the estimate

$$
T_{i}=\left(N_{i}-\frac{1}{2}\right) \Delta t, \quad i=1, \cdots, M .
$$

To estimate the accuracy of the sample mean $\bar{T}$, we compute the empirical standard deviation of the mean by the standard formula

$$
s=\left[\frac{1}{M(M-1)} \sum_{i=1}^{M}\left(T_{i}-\bar{T}\right)^{2}\right]^{1 / 2} .
$$

Using the values

$$
M=500, \quad \Delta t=0.02,
$$

we computed the statistics

$$
\bar{T}=2.3432, \quad s=0.0684 .
$$

The value $\bar{T}$ is within the acceptable probable deviation from the value $T(0,0)=2.3120$ computed from the analytic solution of the boundary value problem.

6. Finite difference methods. In this section we shall describe two finite difference methods for solving the boundary value problem for $T(x, y)$. In later sections we shall state the results of numerical experiments with these methods, establish the convergence of both methods, and define and prove the convergence of iterative schemes for solving the difference equations.

The original boundary value problem for $T(x, y)$ is stated for an infinite domain: $|x| \leqq 1,|y| \leqq \infty$. For numerical purposes we consider a finite subdomain: $|x| \leqq 1,|y| \leqq Y$, where $Y$ is some large number. Picking large integers, $m$ and $n$, we lay down a collection of mesh points

$$
\left(x_{i}, y_{j}\right), \quad i=0, \cdots, m+1, \quad j=-n-1, \cdots, n+1 .
$$

Setting $\Delta x=2 /(m+1)$ and $\Delta y=Y /(n+1)$, we let

$$
x_{i}=-1+i \Delta x, \quad y_{j}=j \Delta y .
$$

The relations between $x$ and $i$, and between $y$ and $j$, are illustrated in Fig. 1 .

6.1. The biased difference method. The partial differential equation $1+y T_{x}+T_{y y} / 2=0$ looks like a heat equation for a "temperature" $T$ in a rod $|y|<Y$ with "time", $x$, propagating to the left in Fig. 1 for $y>0$, but to the right if $y<0$. Accordingly, we may use what is sometimes called the completely implicit difference method. For the heat equation, this method and the balanced method, to be discussed later, are discussed in 


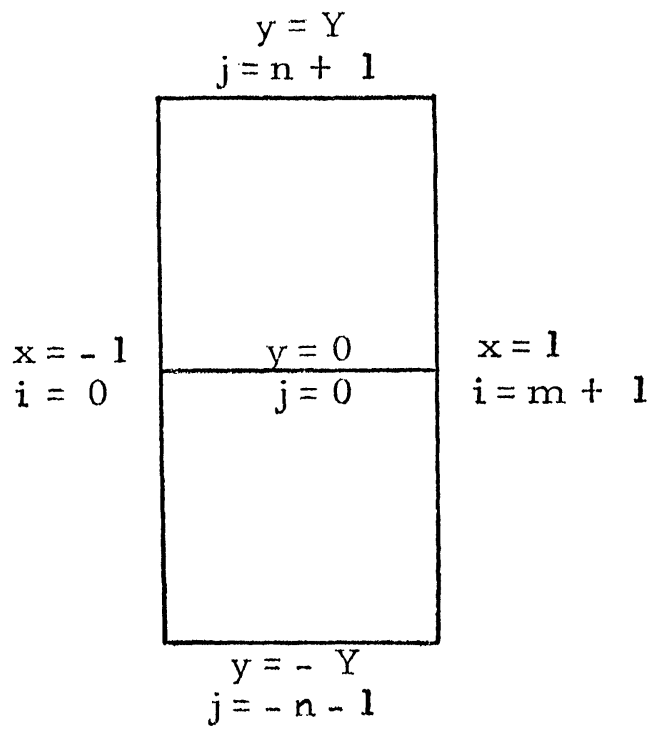

FIG. 1

Richtmyer and Morton [5]. The characteristic patterns of mesh points are illustrated in Fig. 2. If we write $T_{i}{ }^{j}$ as an approximation to $T\left(x_{i}, y_{j}\right)$, the difference equations are

$$
\begin{aligned}
& 1+y_{j} \frac{T_{i+1}^{j}-T_{i}^{j}}{\Delta x}+\frac{1}{2} \frac{T_{i}^{j+1}-2 T_{i}{ }^{j}+T_{i}^{j-1}}{(\Delta y)^{2}}=0 \\
& \begin{aligned}
\text { for } i=0,1, \cdots, m, \quad j=1,2, \cdots, n \\
1+\frac{1}{2} \frac{T_{i}^{1}-2 T_{i}{ }^{0}+T_{i}{ }^{-1}}{(\Delta y)^{2}}=0 \quad \text { for } \quad i=1, \cdots, m ;
\end{aligned}
\end{aligned}
$$$$
1+y_{j} \frac{T_{i+1}^{j}-T_{i}^{j}}{\Delta x}+\frac{1}{2} \frac{T_{i+1}^{j+1}-2 T_{i+1}^{j}+T_{i+1}^{j-1}}{(\Delta y)^{2}}=0
$$

$$
\text { for } i=0,1, \cdots, m, j=-n, \cdots,-1 \text {. }
$$

The boundary conditions for $T_{i}{ }^{j}$ are also illustrated in Fig. 2 . We require (6.4a) $T_{m+1}^{j}=0, j=0, \cdots, n$, and $T_{0}^{j}=0, j=0, \cdots,-n$.

For $y=Y$, we could set $T=0$, but we prefer to use the asymptotic form of the solution. For large $y=Y$, the particle under random acceleration has a large speed to the right, and it may be expected to cross the righthand boundary in a time $=$ distance $/$ speed $=(1-x) / Y$. Thus,

$$
T(x, Y) \cong \frac{1-x}{Y}
$$




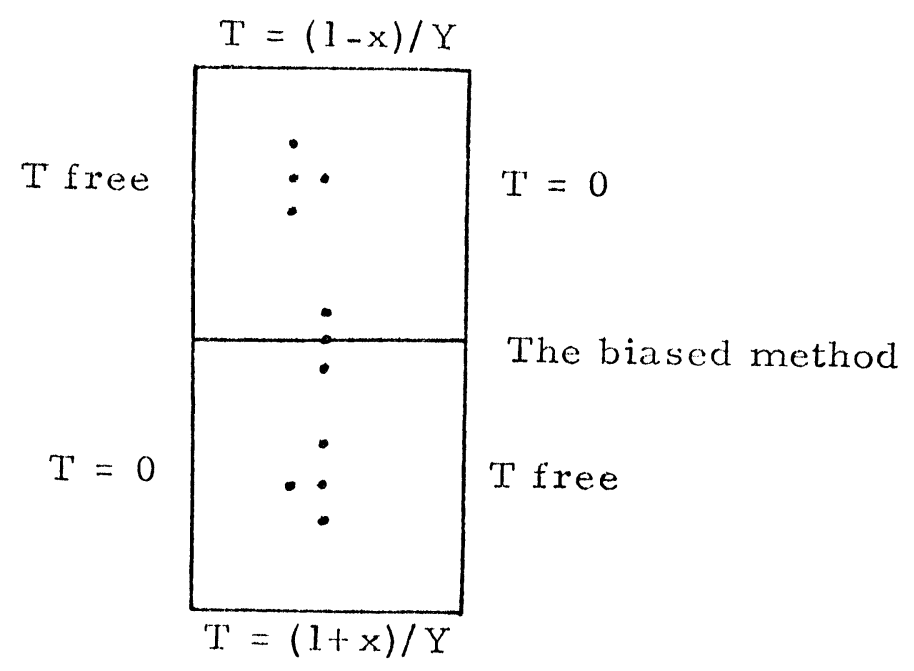

FIG. 2

and similarly

$$
T(x,-Y) \cong \frac{1-x}{Y} .
$$

The asymptotic forms (6.5) also follow from the analytic solution in $\$ 4$ Correspondingly, we set

$$
T_{i}^{n+1}=\frac{1-x_{i}}{Y}, \quad T_{i}^{-n-1}=\frac{1+x_{i}}{Y}, \quad i=0, \cdots, m+1 .
$$

Equations (6.3) and (6.4) define the biased difference method.

6.2. The balanced difference method. We define another difference scheme by the characteristic mesh pattern

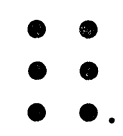

This mesh pattern, when it is applied to the heat equation, yields the Crank-Nicholson method [5]. Using the abbreviations

$$
T_{i+1 / 2}^{j} \equiv \frac{1}{2}\left(T_{i}^{j}+T_{i+1}^{j}\right), \quad \delta^{2} T_{i+1 / 2}^{j} \equiv T_{i+1 / 2}^{j+1}-2 T_{i+1 / 2}^{j}+T_{i+1 / 2}^{j-1},
$$

we define the balanced method by the difference equations

$$
\begin{aligned}
& 1+y_{j} \frac{T_{i+1}^{j}-T_{i}^{j}}{\Delta x}+\frac{1}{2} \frac{\delta^{2} T_{i+1 / 2}^{j}}{(\Delta y)^{2}}=0 \\
& \quad \text { for } \quad i=0, \cdots, m, j=-n, \cdots, n .
\end{aligned}
$$


For $j=0$, we shall later require a stronger condition, to be given in formula (6.8b). In a closed interior subdomain the biased method has truncation error $O\left(\Delta x+(\Delta y)^{2}\right)$, whereas the balanced method has truncation error $O\left((\Delta x)^{2}+(\Delta y)^{2}\right)$. Unfortunately, as we shall note later, these estimates do not hold near the points $x= \pm 1, y=0$.

For $x= \pm 1$, we require (6.9a) $\quad T_{m+1}^{j}=0, \quad j=1, \cdots, n$, and $T_{0}^{j}=0, j=-1, \cdots,-n$. Note that we do not require $T_{m+1}^{0}=0$ and $T_{0}{ }^{0}=0$, as we did in (6.4a) for the biased method. For $y= \pm Y$ we again use the asymptotic boundary values

$$
T_{i}{ }^{n+1}=\frac{1-x_{i}}{Y}, \quad T_{i}^{-n-1}=\frac{1+x_{i}}{Y}, \quad i=0, \cdots, m+1 .
$$

In the biased method, formula (6.3) presents $2 n(m+1)+m$ equations for the unknowns $T_{i}^{j}$ with

$$
j= \begin{cases}1, \cdots, n & \text { for } i=0, \cdots, m \\ 0 & \text { for } i=1, \cdots, m, \\ -1, \cdots,-n & \text { for } i=1, \cdots, m+1 .\end{cases}
$$

In the balanced method, formula (6.8a) presents $(m+1)(2 n+1)$ equations for the unknowns $T_{i}{ }^{j}$ with

$$
j= \begin{cases}1, \cdots, n & \text { for } i=0, \cdots, m, \\ 0 & \text { for } i=0, \cdots, m+1, \\ -1, \cdots,-n & \text { for } i=1, \cdots, m+1 .\end{cases}
$$

Formula (6.11) contains $2 n(m+1)+(m+2)$ pairs of indices. Thus, we have one more unknown than we have equations. To remedy this defect, we replace (6.8a) for $j=0$ by the equations

$$
1+\frac{1}{2} \frac{\delta^{2} T_{i}^{0}}{(\Delta y)^{2}}=0, \quad i=0, \cdots, m+1 .
$$

This formula implies formula (6.8a) or $j=0$; but the converse is not true.

6.3. The balanced method with averaging. We shall see later that the balanced method yields numbers $T_{i}{ }^{j}$ which are not very good approximations to the true values $T\left(x_{i}, y_{j}\right)$. However, the average values $T_{i+1 / 2}^{j}$ $\equiv\left(T_{i}{ }^{j}+T_{i+1}^{j}\right) / 2$ provide excellent approximations to the true values $T\left(x_{i+1 / 2}, y_{j}\right)$.

7. Numerical experience. From the analytic solution for $y=0$,

$$
\begin{aligned}
T(x, 0)=\frac{3^{11 / 6}}{2 \Gamma(1 / 3)}\left(1-x^{2}\right)^{1 / 6}\left[F \left(-\frac{1}{3}, 1, \frac{7}{6},\right.\right. & \left.\frac{1-x}{2}\right) \\
& \left.+F\left(-\frac{1}{3}, 1, \frac{7}{6}, \frac{1+x}{2}\right)\right],
\end{aligned}
$$


TABLE 1

\begin{tabular}{c|c|c|c}
\hline$x$ & Analytic $T(x, 0)$ & Biased $T(x, 0)$ & $\begin{array}{c}\text { Balanced without } \\
\text { averaging } T(x, 0)\end{array}$ \\
\cline { 1 - 2 } 0.0 & 2.3120 & 2.1513 & 2.4750 \\
0.2 & 2.289 & 2.125 & 2.115 \\
0.4 & 2.216 & 2.042 & 2.409 \\
0.6 & 2.076 & 1.878 & 1.850 \\
0.8 & 1.813 & 1.554 & 2.171 \\
\hline
\end{tabular}

TABLE 2

\begin{tabular}{c|c|c}
\hline$x$ & Analytic $T(x, 0)$ & Balanced with averaging $T(x, 0)$ \\
\hline 0 & 2.3120 & 2.3192 \\
$2 / 11$ & 2.293 & 2.268 \\
$4 / 11$ & 2.233 & 2.246 \\
$6 / 11$ & 2.123 & 2.088 \\
$8 / 11$ & 1.933 & 1.980 \\
$10 / 11$ & 1.550 & 1.432 \\
\hline
\end{tabular}

it is easy to compute exact values. We shall use these values to check the biased method and the balanced method with and without averaging. The best method appears to be the balanced method with averaging. The biased method is less accurate, and the balanced method without averaging is the least accurate. Since $T(x, y) \equiv T(-x,-y)$, we only record the results for $0 \leqq x<1$.

For the biased method and for the balanced method without averaging we took

$$
Y=20, \quad \Delta x=2 / 10, \quad \Delta y=20 / 101 .
$$

The results, along with the values of $T(x, 0)$ from the analytic solution, are given in Table 1.

For the balanced method with averaging we took

$$
y=20, \quad \Delta x=2 / 11, \quad \Delta y=20 / 101 .
$$

Here the values were computed at the midpoints $x_{i+1 / 2}$. The results are given in Table 2.

8. Convergence of the biased method. Convergence is hard to prove for our problem because, as the mesh is refined, the truncation error does not tend to zero. In [9] Cannon and Hill give a proof of convergence for the biased method which assumes that the true solution $T(x, y)$ has continuous derivatives of fourth order in the closed domain $|x| \leqq 1,|y| \leqq Y$. 


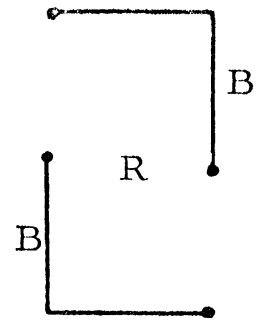

FIG. 3

An examination of the analytic solution derived in $\$ 4$ shows that, for $x$ near $\pm 1, T(x, 0)$ behaves like $\left(1-x^{2}\right)^{1 / 6}$. Thus, $T(x, y)$ does not even have bounded derivatives of first order in the closed domain.

Let $L$ be the differential operator

$$
L=-y \frac{\partial}{\partial x}-\frac{1}{2} \frac{\partial^{2}}{\partial y^{2}}
$$

and let $L_{h}$ be the corresponding difference operator for the biased method described in $\S 6$, where $h=\Delta x$ and where $\Delta y$ is some function of $h$ which tends to zero as $h \rightarrow 0$. In this notation, the true solution $T(x, y)$ and the approximate solution $T_{i}{ }^{j}$ satisfy the equations

$$
L T(x, y)=1, \quad L_{h} T_{i}{ }^{j}=1 .
$$

For a rectangle $|x| \leqq 1,|y| \leqq Y$, we define the boundary set $B$ to be the points $(x, y)$ for which $y= \pm Y$, or for which $x=1$ and $y \geqq 0$, or for which $x=-1$ and $y \leqq 0$. See Fig. 3 . We define the set $B_{h}$ to consist of the mesh points $\left(x_{i}, y_{j}\right)$ lying on $B$.

Cannon and Hill showed that $L_{h}{ }^{-1}$ is a positive operator: If $L_{h} u_{i}{ }^{j} \geqq 0$ in the rectangle, and if $u_{i}{ }^{j} \geqq 0$ in $B_{h}$, then $u_{i}{ }^{j} \geqq 0$ in the rectangle. In fact, if $u_{i}{ }^{j}$ attains a negative minimum, and if $\left(x_{i}, y_{j}\right)$ is a point with maximum $y_{j}$ at which the minimum $u_{i}^{j}$ is attained, then $u_{i}{ }^{j+1}>u_{i}{ }^{j}$, and the difference equations yield a contradiction.

For example, if $y_{j}<0$, we would have

$$
L_{h} u_{i-1}^{j} \equiv-y_{j} \frac{u_{i}{ }^{j}-u_{i-1}^{j}}{\Delta x}-\frac{1}{2} \frac{u_{i}^{j+1}-2 u_{i}^{j}+u_{i}{ }^{j-1}}{(\Delta y)^{2}}<0,
$$

which contradicts the assumption $L_{h} u \geqq 0$. Exactly this line of reasoning has been used to prove the positivity of the inverse operator for the completely implicit difference method for the heat equation; cf. [5, p. 48].

We now use majorization to conclude that

$$
\left|u_{i}{ }^{j}\right| \leqq \delta_{B}+\delta_{L}\left(Y^{2}-y_{j}{ }^{2}\right)
$$


if $\left|L_{h} u_{i}{ }^{j}\right| \leqq \delta_{L}$ in the rectangle and if $\left|u_{i}{ }^{j}\right| \leqq \delta_{B}$ on $B_{h}$. In fact, since $L_{h}\left(y_{j}^{2}\right)=-1$, the numbers

$$
v_{i}{ }^{j}=u_{i}^{j}+\delta_{B}+\delta_{L}\left(Y^{2}-y_{j}^{2}\right)
$$

are greater than or equal to 0 on $B_{h}$, and $L_{h} v_{i}{ }^{j} \geqq 0$. Therefore, $v_{i}{ }^{j} \geqq 0$ in the rectangle. The same is true of the numbers $w_{i}^{j}$ formed by replacing $u_{i}^{j}$ by $-u_{i}{ }^{j}$ in the expression for $v_{i}{ }^{j}$; and (8.3) follows.

Incidentally, the inequality (8.3) shows that the original difference equations for $T_{i}{ }^{j}$ have one and only one solution. Otherwise, by the alternative of linear algebra, there would be a vector $\left(u_{i}^{j}\right) \neq 0$ with $L_{h} u_{i}^{j}=0$, and with $u_{i}^{j}=0$ on $B_{h}$. Taking $\delta_{L}=\delta_{B}=0$ in (8.3), we have a contradiction.

We are now ready to prove convergence. Given $\epsilon>0$, we shall show that there exists a number $Y_{0}(\epsilon)$ so large that, if $Y \geqq Y_{0}(\epsilon)$, and if $h<h_{0}(\epsilon, Y)$, then $\left|T_{i}{ }^{j}-T\left(x_{i}, y_{j}\right)\right|<4 \epsilon$ at all mesh points in the rectangle $|x| \leqq 1,|y| \leqq Y$.

We pick $Y_{0}=Y_{0}(\epsilon)$ so large that, for $|x| \leqq 1$,

$$
\left|T(x, Y)-\frac{1-x}{Y}\right|<\epsilon \text { and }\left|T(x,-Y)-\frac{1+x}{Y}\right|<\epsilon
$$

if $Y \geqq Y_{0}$. Now let $Y$ be fixed at any value greater than or equal to $Y_{0}$. In the rest of the proof we are concerned only with the convergence of $T_{i}{ }^{j}$ to $T\left(x_{i}, y_{j}\right)$ in the bounded rectangle $|x| \leqq 1,|y| \leqq Y$.

By definition, the truncation error is

$$
L_{h} T\left(x_{i}, y_{j}\right)-L T\left(x_{i}, y_{j}\right)=L_{h} T\left(x_{i}, y_{j}\right)-1 .
$$

Unfortunately, the truncation error does not tend to zero as the mesh parameter $h \rightarrow 0$ because $T(x, y)$ is not sufficiently smooth in the closed rectangle. We remedy this difficulty by finding a family of functions $T(x, y, a)$ with the properties

$$
\begin{aligned}
& T(x, y, a) \text { is smooth for }|x| \leqq 1, \quad|y| \leqq Y \quad \text { if } a>1 \\
& T(x, y, a) \rightarrow T(x, y) \text { as } a \rightarrow 1+0 \\
& L T(x, y, a) \rightarrow L T(x, y) \text { as } a \rightarrow 1+0 .
\end{aligned}
$$

Such functions $T(x, y, a)$ are given by the similar solutions

$$
T(x, y, a)=a^{2 / 3} T\left(a^{-1} x, a^{-1 / 3} y\right), \quad a>1,
$$

constructed in $\S 2$. We have smoothness because $\left|a^{-1} x\right| \leqq a^{-1}<1$ for $|x| \leqq 1$. We have $T(x, y, a) \rightarrow T(x, y)$ by the continuity of $T(x, y)$ in the closed rectangle. And for (8.7) we even have $\operatorname{LT}(x, y, a)=1=\operatorname{LT}(x, y)$ for each $a>1$. 
We pick $a>1$ so small that, for $|x| \leqq 1$ and $|y| \leqq Y$,

$$
|T(x, y, a)-T(x, y)|<\epsilon .
$$

Having fixed $a$, we pick $h_{0}$ so small that, for all mesh points,

$$
\left|L_{h} T\left(x_{i}, y_{j}, a\right)-L T\left(x_{i}, y_{j}, a\right)\right|<\epsilon Y^{-2}
$$

for all $h \leqq h_{0}$. This is possible because $T(x, y, a)$ has derivatives of all orders in the closed rectangle.

We now define

$$
u_{i}^{j}=T_{i}{ }^{j}-T\left(x_{i}, y_{j}, a\right) .
$$

On the boundary $B_{h}$, we have, by (8.4) and (8.9),

$$
\left|u_{i}^{j}\right| \leqq \delta_{B}=\epsilon+\epsilon=2 \epsilon \text { on } B_{h} .
$$

Moreover, we have

$$
\begin{aligned}
\left|L_{h} u_{i}{ }^{j}\right| & =\left|L_{h} T_{i}^{j}-L_{h} T\left(x_{i}, y_{j}, a\right)\right| \\
& =\left|1-L_{h} T\left(x_{i}, y_{j}, a\right)\right| \\
& =\left|L T\left(x_{i}, y_{j}, a\right)-L_{h} T\left(x_{i}, y_{j}, a\right)\right|<\epsilon Y^{-2} .
\end{aligned}
$$

Taking $\delta_{L}=\epsilon Y^{-2}$ in the inequality (8.3), we find

$$
\left|u_{i}^{j}\right| \leqq \delta_{B}+\delta_{L}\left(Y^{2}-y_{j}^{2}\right) \leqq 3 \epsilon .
$$

We now find

$$
\begin{aligned}
\left|T_{i}{ }^{j}-T\left(x_{i}, y_{j}\right)\right| & \leqq\left|T_{i}{ }^{j}-T\left(x_{i}, y_{j}, a\right)\right|+\left|T\left(x_{i}, y_{j}, a\right)-T\left(x_{i}, y_{j}\right)\right| \\
& \leqq 3 \epsilon+\epsilon=4 \epsilon .
\end{aligned}
$$

9. Convergence of the balanced method. In this section we shall prove convergence in the mean for the balanced method with averaging. For a rectangle $|x| \leqq 1,|y| \leqq Y$ and for a mesh defined as in $\S 6$, we define the inner product $(u, v)$ and the norm $\|u\|$ for mesh functions $u_{i}{ }^{j}, v_{i}{ }^{j}$ as

$$
\begin{aligned}
(u, v) & =\sum_{i=0}^{m} \sum_{j=n}^{n}\left(u_{i}^{j}\right)\left(v_{i}^{j}\right) \Delta x \Delta y, \\
\|u\| & =(u, u)^{1 / 2} .
\end{aligned}
$$

Let $h$ be a mesh parameter, say $h=\Delta x$; and let $\Delta y$ be some function of $h$ which tends to zero as $h \rightarrow 0$. Typically, $\Delta y=$ some constant times $h$. Let $L$ be the differential operator

$$
L=-y \frac{\partial}{\partial x}-\frac{1}{2} \frac{\partial^{2}}{\partial y^{2}}
$$


and let $L_{h}$ be the corresponding difference operator for the balanced method defined in $\S 6$. Thus,

$$
L T(x, y)=1 \text { and } L_{h} T_{i}{ }^{j}=1,
$$

where $T(x, y)$ is the true solution of the boundary value problem, and where $T_{i}{ }^{j}$ is the balanced difference solution satisfying the approximate boundary conditions prescribed in formulas (6.9). Given $\epsilon>0$, we shall show that there is a number $Y_{0}(\epsilon)$ such that, if $Y \geqq Y_{0}(\epsilon)$, and if $h \leqq h_{0}(\epsilon, Y)$, then

$$
\left\|T_{i+1 / 2}^{j}-T\left(x_{i+1 / 2}, y_{j}\right)\right\|<18 \epsilon .
$$

Here we have used the abbreviation $T_{i+1 / 2}^{j} \equiv \frac{1}{2}\left(T_{i+1}^{j}+T_{i}^{j}\right)$. But by $T\left(x_{i+1 / 2}, y_{j}\right)$ we mean the exact value of $T(x, y)$ at the point $\left(x_{i}+(\Delta x) / 2, y_{j}\right)$.

Notation. If $u_{i}{ }^{j}$ is given for $i=0, \cdots, m+1$ and for $|j| \leqq n+1$, we define

$$
\begin{aligned}
& u_{i+1 / 2}^{j}=\frac{1}{2}\left(u_{i+1}^{j}+u_{i}^{j}\right), \quad i=0,1, \cdots, m, \quad|j| \leqq n+1, \\
& \Delta u_{i+1 / 2}^{j}=u_{i+1 / 2}^{j+1}-u_{i+1 / 2}^{j}, \quad i=0,1, \cdots, m, \quad j=-n-1, \cdots, n, \\
& \nabla u_{i+1 / 2}^{j}=u_{i+1 / 2}^{j}-u_{i+1 / 2}^{j-1}, \quad i=0,1, \cdots, m, \quad j=-n, \cdots, n+1, \\
& \delta^{2} u_{i+1 / 2}^{j}=u_{i+1 / 2}^{j+1}-2 u_{i+1 / 2}^{j}+u_{i+1 / 2}^{j-1}, \quad i=0,1, \cdots, m,|j| \leqq n, \\
& M_{h} u_{i}{ }^{j}=-y_{j} \frac{u_{i+1}^{j}-u_{i}^{j}}{\Delta x}-\frac{1}{2} \frac{\delta^{2} u_{i+1 / 2}^{j}}{(\Delta y)^{2}}, \quad i=0,1, \cdots, m, \quad|j| \leqq n,
\end{aligned}
$$

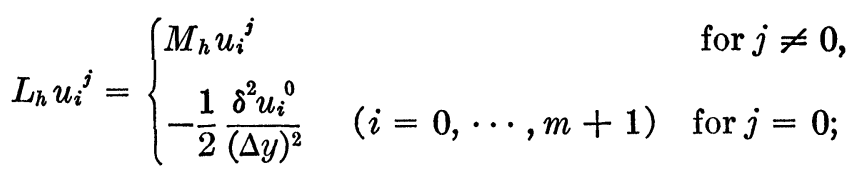

$$
\begin{aligned}
& B=\text { the set of points }(x, y) \text { with } y= \pm Y \\
& \text { or with } x=1 \text { and } y>0 \\
& \text { or with } x=-1 \text { and } y<0 \text {, } \\
& B_{h}=\text { the mesh points }\left(x_{i}, y_{j}\right) \text { on } B \text {, } \\
& \beta\left(u_{i}{ }^{j}\right)=\max \left|u_{i}^{j}\right| \text { for }\left(x_{i}, y_{j}\right) \text { in } B_{h} \text {. }
\end{aligned}
$$

We shall first prove an inequality which shows that $\left\|u_{i+1 / 2}^{j}\right\|$ is small if $\left\|M_{h} u_{i}{ }^{j}\right\|$ is small and if $\beta\left(u_{i}{ }^{j}\right)$ is small. We shall prove that

$$
\left\|u_{i+1 / 2}^{j}\right\|^{2} \leqq Y\left(8+Y^{3}\right) \beta^{2}\left(u_{i}^{j}\right)+4 Y^{4}\left\|M_{h} u_{i}^{j}\right\|^{2} .
$$


From the inequality (9.5), the existence and uniqueness of the solution $T_{i}{ }^{j}$ to $L_{h} T_{i}{ }^{j}=1$, with given values for $T_{i}{ }^{j}$ on $B_{h}$, follow from the alternative of linear algebra: Otherwise, there would be some nonzero vector $u_{i}{ }^{j}$ with $\beta\left(u_{i}{ }^{j}\right)=0$ and with $L_{h} u_{i}{ }^{j} \equiv 0$. But $L_{h} u_{i}{ }^{j} \equiv 0$ implies $M_{h} u_{i}{ }^{j} \equiv 0$, and then (9.5) implies $u_{i+1 / 2}^{j}=0$ for $i=0, \cdots, m$ and $|j| \leqq n$. Now $\beta=0$ implies $u_{i}{ }^{j}=0$ for all $j \neq 0$, since

$$
\begin{aligned}
& \frac{1}{2} u_{i}^{j}=\sum_{k=i}^{m}(-)^{k-i} u_{k+1 / 2}^{j} \pm \frac{1}{2} u_{m+1}^{j}=0, \quad j>0, \quad i \geqq 0, \\
& \frac{1}{2} u_{i}^{j}=\sum_{k=0}^{i-1}(-)^{i-k} u_{k+1 / 2}^{j} \pm \frac{1}{2} u_{0}^{j}=0, \quad j<0, \quad i \leqq m+1 .
\end{aligned}
$$

Now we use the equation $L u_{i}^{0}=0$ to obtain

$$
u_{i}^{0}=\frac{1}{2}\left(u_{i}^{1}+u_{i}^{(-1)}\right)=0, \quad i=0,1, \cdots, m+1 .
$$

Hence, the vector $u_{i}^{j}$ is zero.

Now we shall prove the inequality (9.5). Let $w^{j}=y_{j}{ }^{2}-Y^{2}$. Then $\delta^{2} w^{j}=2(\Delta y)^{2}$, and $w^{ \pm(n+1)}=0$. Therefore,

$$
\begin{aligned}
2(\Delta y)^{2} \sum_{j=-n}^{n}\left(u_{i+1 / 2}^{j}\right)^{2} & =\sum_{j=-n}^{n}\left(u_{i+1 / 2}^{j}\right)^{2}\left(w^{j+1}-2 w^{j}+w^{j-1}\right) \\
& =\gamma_{i}+\sum_{j=-n}^{n} w^{j} \delta^{2}\left[\left(u_{i+1 / 2}^{j}\right)^{2}\right],
\end{aligned}
$$

where the boundary term

$$
\gamma_{i}=-w^{-n}\left(u_{i+1 / 2}^{-n-1}\right)^{2}-w^{n}\left(u_{i+1 / 2}^{n+1}\right)^{2} .
$$

But the difference operators $\delta^{2}, \Delta$, and $\nabla$ satisfy

$$
\delta^{2}\left[\left(u^{j}\right)^{2}\right]=2 u^{j} \delta^{2} u^{j}+\left(\Delta u^{j}\right)^{2}+\left(\nabla u^{j}\right)^{2} .
$$

Since $\delta^{2} u_{i+1 / 2}^{j}$ is related to $M_{h} u_{i+1 / 2}^{j}$ by the identity

$$
-\frac{1}{2(\Delta y)^{2}} \delta^{2} u_{i+1 / 2}^{j}=M_{h} u_{i}^{j}+\frac{y_{j}}{\Delta x}\left(u_{i+1}^{j}-u_{i}^{j}\right),
$$

we find, using $u_{i+1 / 2}\left(u_{i+1}-u_{i}\right)=\left\{\left(u_{i+1}\right)^{2}-\left(u_{i}\right)^{2}\right\} / 2$,

$$
2 u_{i+1 / 2}^{j} \delta^{2} u_{i+1 / 2}^{j}=-4(\Delta y)^{2}\left[u_{i+1 / 2}^{j} M_{h} u_{i}^{j}+\frac{y_{j}}{2 \Delta x}\left\{\left(u_{i+1}^{j}\right)^{2}-\left(u_{i}^{j}\right)^{2}\right\}\right] .
$$

Since $w^{j}=\left(y_{j}\right)^{2}-Y^{2} \leqq 0$, we have from (9.8),

$$
w^{j} \delta^{2}\left[\left(u_{i+1 / 2}^{j}\right)^{2}\right] \leqq 2 w^{j} u_{i+1 / 2}^{j} \delta^{2} u_{i+1 / 2}^{j} .
$$


Now (9.6) yields

$$
\begin{aligned}
2(\Delta y)^{2} \sum_{j=-n}^{n}\left(u_{i+1 / 2}^{j}\right)^{2} \leqq & \gamma_{i}+2 \sum_{j=-n}^{n} w^{j} u_{i+1 / 2}^{j} \delta^{2} u_{i+1 / 2}^{j} \\
\leqq & \gamma_{i}-4(\Delta y)^{2} \sum_{j=-n}^{n} w^{j} u_{i+1 / 2}^{j} M_{h} u_{i+1 / 2}^{j} \\
& -2 \frac{(\Delta y)^{2}}{\Delta x} \sum_{j=-n}^{n} w^{j} y_{j}\left\{\left(u_{i+1}^{j}\right)^{2}-\left(u_{i}^{j}\right)^{2}\right\} .
\end{aligned}
$$

If we sum this inequality for $i=0, \cdots, m$ and multiply by $\Delta x$, we find, using the inner product notation (9.1),

$$
\begin{aligned}
2 \Delta y\left\|u_{i+1 / 2}^{j}\right\|^{2} \leqq \sum_{i=0}^{m} \gamma_{i} \Delta x- & 4 \Delta y\left(w^{j} u_{i+1 / 2}^{j}, M_{h} u_{i+1 / 2}^{j}\right) \\
& -2(\Delta y)^{2} \sum_{j=-n}^{n} w^{j} y_{j}\left\{\left(u_{m+1}^{j}\right)^{2}-\left(u_{0}^{j}\right)^{2}\right\} .
\end{aligned}
$$

Since $w^{j} \leqq 0$, we have

$$
-\sum_{j=-n}^{n} w^{j} y_{j}\left\{\left(u_{m+1}^{j}\right)^{2}-\left(u_{0}^{j}\right)^{2}\right\} \leqq \sum_{j=1}^{m}\left(-w_{j}\right) y_{j}\left(u_{m+1}^{j}\right)^{2}+\sum_{j=-1}^{-n} w_{j} y_{j}\left(u_{0}^{j}\right)^{2} .
$$

Since $w^{ \pm n}=\left(y_{n}\right)^{2}-Y^{2}=(Y-\Delta y)^{2}-Y^{2}=-2 Y \Delta y+(\Delta y)^{2}$, we have from (9.7):

$$
\gamma_{i}=2(\Delta y)\left(Y-\frac{\Delta y}{2}\right)\left[\left(u_{i+1 / 2}^{-n-1}\right)^{2}+\left(u_{i+1 / 2}^{n+1}\right)^{2}\right] .
$$

Division of formula (9.9) by $2 \Delta y$ now yields

$$
\begin{array}{r}
\left\|u_{i+1 / 2}^{j}\right\|^{2} \leqq\left(Y-\frac{\Delta y}{2}\right) \sum_{i=0}^{m}\left[\left(u_{i+1 / 2}^{-n-1}\right)^{2}+\left(u_{i+1 / 2}^{n+1}\right)^{2}\right] \Delta x \\
-2\left(w^{j} u_{i+1 / 2}^{j}, M_{h} u_{i+1 / 2}^{j}\right)+\Delta y \sum_{j=1}^{n}\left(-w_{j}\right) y_{j}\left(u_{m+1}^{j}\right)^{2} \\
+\Delta y \sum_{j=-1}^{-n} w_{j} y_{j}\left(u_{0}^{j}\right)^{2} .
\end{array}
$$

Formula (9.10) is a difference analogue on the inequality of Fichera:

$$
\begin{aligned}
& \int_{-1}^{1} \int_{-Y}^{Y} u^{2} d x d y \leqq Y \int_{-1}^{1}\left[u^{2}(-Y, x)+u^{2}(Y, x)\right] d x \\
& \quad-2 \int_{-1}^{1} \int_{-Y}^{Y}\left(y^{2}-Y^{2}\right) u \cdot\left(-y u_{x}-\frac{1}{2} u_{y y}\right) d x d y \\
& +\int_{0}^{Y}\left(Y^{2}-y^{2}\right) y u^{2}(1, y) d y+\int_{-Y}^{0}\left(Y^{2}-y^{2}\right)(-y) u^{2}(-1, y) d y
\end{aligned}
$$

which holds for every function $u(x, y)$ in $C^{2}$. 
Since the boundary sums in (9.10) contain only points of $B_{h}$, on which we assume $\left|u_{i}^{j}\right| \leqq \beta$, we find

$$
\left\|u_{i+1 / 2}^{j}\right\|^{2} \leqq 4 Y \beta^{2}-2\left(w^{j} u_{i+1 / 2}^{j}, M_{h} u_{i}^{j}\right)+2 \beta^{2} \Delta y \sum_{j=1}^{n}\left(Y^{2}-y_{j}^{2}\right) y_{j} .
$$

But

$$
\Delta y \sum_{j=1}^{n}\left(Y^{2}-y_{j}^{2}\right) y_{j}<\int_{0}^{Y}\left(Y^{2}-y^{2}\right) y d y=\frac{1}{4} Y^{4}
$$

since the sum on the left is the trapezoidal sum approximating the integral on the right, in which the integrand has a negative second derivative. Moreover,

$$
\begin{aligned}
-2\left(w^{j} u_{i+1 / 2}^{j}, M_{h} u_{i}^{j}\right) & =\left(u_{i+1 / 2}^{j},-2 w^{j} M_{h} u_{i}^{j}\right) \\
& \leqq \frac{1}{2}\left\|u_{i+1 / 2}^{j}\right\|^{2}+2 Y^{4}\left\|M_{h} u_{i}^{j}\right\|^{2}
\end{aligned}
$$

since $(a, b) \leqq \frac{1}{2}\left(\|a\|^{2}+\|b\|^{2}\right)$, and since $\left|w^{j}\right| \leqq Y^{2}$. The last three inequalities yield

$$
\left\|u_{i+1 / 2}^{j}\right\|^{2}<4 Y \beta^{2}+\frac{1}{2}\left\|u_{i+1 / 2}^{j}\right\|^{2}+2 Y^{4}\left\|M_{h} u_{i}^{j}\right\|^{2}+\frac{1}{2} \beta^{2} Y^{4},
$$

which implies the required inequality (9.5).

It is now easy to complete the proof of convergence in the mean. Choose $\epsilon>0$. Now choose $Y_{0}=Y_{0}(\epsilon) \geqq 1$ so large that, for all $Y \geqq Y_{0}$,

$$
\left|T(x, \pm Y)-\frac{1 \mp x}{Y}\right|<\epsilon Y^{-2} \text { for }|x| \leqq 1 .
$$

It is possible to do this because of the asymptotic formula

$$
T(x, \pm Y)-\frac{1 \mp x}{Y}=O\left(Y^{-4}\right) \quad \text { as } \quad Y \rightarrow \infty
$$

which holds for the true solution $T(x, y)$ derived in $\$ 4$. Now let $Y$ be fixed at some value greater than or equal to $Y_{0}$. As in the preceding section, choose $a$ so near $1+0$ that the similar solution $T(x, y, a)$ satisfies

$$
|T(x, y, a)-T(x, y)|<\epsilon / Y^{2}, \quad|x| \leqq 1, \quad|y| \leqq Y .
$$

Since $T(x, y, a)$ is smooth, and since $L T(x, y, a)=1$, we can choose $h_{0}$ so small that, for all mesh points in the rectangle,

$$
\left|L_{h} T\left(x_{i}, y_{j}, a\right)-1\right|<\epsilon / Y^{3}
$$

if $h<h_{0}(\epsilon, Y)$. We can also choose $h_{0}$ so small that

$$
\left|\left[T\left(x_{i}, y_{j}, a\right)+T\left(x_{i+1}, y_{j}, a\right)\right] / 2-T\left(x_{i+1 / 2}, y_{j}, a\right)\right|<\epsilon / Y .
$$


Having fixed $Y, a$ and $h$, we define

$$
u_{i}{ }^{j}=T_{i}{ }^{j}-T\left(x_{i}, y_{j}, a\right), \quad i=0,1, \cdots, m+1, \quad|j| \leqq n+1,
$$

where $T_{i}{ }^{j}$ is the solution of the balanced difference problem. We then have the identity, for $i=0,1, \cdots, m$ and $|j| \leqq n+1$,

$$
\begin{aligned}
T_{i+1 / 2}^{j}-T\left(x_{i+1 / 2}, y_{j}\right) & \\
= & u_{i+1 / 2}^{j}+\left[T\left(x_{i}, y_{j}, a\right)+T\left(x_{i+1}, y_{j}, a\right)\right] / 2 \\
& -T\left(x_{i+1 / 2}, y_{j}, a\right)+T\left(x_{i+1 / 2}, y_{j}, a\right) \\
& -T\left(x_{i+1 / 2}, y_{j}\right) .
\end{aligned}
$$

For any vector $v_{i}^{j}$ we have

$$
\left\|v_{i}^{j}\right\|=\left[\sum_{i=0}^{m} \sum_{j=-n}^{n}\left(v_{i}{ }^{j}\right)^{2} \Delta x \Delta y\right]^{1 / 2} \leqq 4 Y \max \left|v_{i}{ }^{j}\right|
$$

since $(m+1) \Delta x=2$ and $(2 n+1) \Delta y=2 Y-\Delta y$. From (9.17), (9.19) and (9.21), we now conclude

$$
\begin{aligned}
\left\|T_{i+1 / 2}^{j}-T\left(x_{i+1 / 2}, y_{j}\right)\right\| & \leqq\left\|u_{i+1 / 2}^{j}\right\|+4 Y\left(\epsilon / Y+\epsilon / Y^{2}\right) \\
& \leqq\left\|u_{i+1 / 2}^{j}\right\|+8 \epsilon .
\end{aligned}
$$

Now we only have to bound $\left\|u_{i+1 / 2}^{j}\right\|$. On the boundary $B_{h}$, by (9.15) and (9.17),

$$
\beta \equiv \max \left|u_{i}{ }^{j}\right|<2 \epsilon / Y^{2} .
$$

Moreover, for $0 \leqq i \leqq m$ and $|j| \leqq n$, we have

$$
L_{h} T_{i}{ }^{j}=M_{h} T_{i}{ }^{j}=1
$$

and, by (9.18),

$$
\left|M_{h} T\left(x_{i}, y_{j}, a\right)-1\right|<\epsilon / Y^{3} .
$$

Therefore, $\left|M_{h} u_{i}^{j}\right|<\epsilon / Y^{3}$, and

$$
\left\|M_{h} u_{i}^{j}\right\|<4 \epsilon / Y^{2} .
$$

The inequality (9.5) now yields

$$
\begin{aligned}
\left\|u_{i+1 / 2}^{j}\right\|^{2} & \leqq Y\left(8+Y^{3}\right)\left(2 \epsilon / Y^{2}\right)^{2}+4 Y^{4}\left(4 \epsilon / Y^{2}\right)^{2} \\
& \leqq 9 \cdot 4 \epsilon^{2}+4 \cdot 16 \epsilon^{2}=100 \epsilon^{2} .
\end{aligned}
$$

From (9.23) we now find the required inequality (9.3). This completes the proof of convergence in the mean for the balanced difference scheme with averaging. 
10. Solution of the biased difference equations. In the biased method, which is described in $\$ 6$, we have to solve $2 n(m+1)+m$ inhomogeneous linear equations for an equal number of unknowns $T_{i}{ }^{j}$. If the values $T_{i}{ }^{0}$, corresponding to $y=0$, were known, the values $T_{i}{ }^{j}$ for $j>0$ could be calculated by solving the completely implicit parabolic difference equations

$$
1+y_{j} \frac{T_{i+1}^{j}-T_{i}^{j}}{\Delta x}+\frac{1}{2} \frac{T_{i}^{j+1}-2 T_{i}^{j}+T_{i}^{j-1}}{(\Delta y)^{2}}=0, \quad j>0 .
$$

The values $T_{m+1}^{j}=0, j>0$, are given, and the values $T_{i}{ }^{n+1}=\left(1-x_{i}\right) / Y$ are also given. The implicit equations (10.1) are first solved for $i=m$, $j=1,2, \cdots, n$. Then they are solved for $i=m-1, j=1,2, \cdots, n$, etc., until finally they are solved for $i=0, j=1,2, \cdots, n$. The technique of doing this is described in Richtmyer and Morton [5, p. 200]. Similarly, the equations

$$
1+y_{j} \frac{T_{i+1}^{j}-T_{i}^{j}}{\Delta x}+\frac{1}{2} \frac{T_{i+1}^{j+1}-2 T_{i+1}^{j}+T_{i+1}^{j-1}}{(\Delta y)^{2}}=0, \quad j<0,
$$

could be solved. But now we would proceed from left to right: $i=0,1, \cdots, m$. Of course, owing to the symmetry to our particular problem, we would not need to calculate $T_{i}{ }^{j}$ for $j<0$, since $T_{i}{ }^{j}=T_{m+1-i}^{-j}$. Thus the problem is solved if we can find the central values $T_{i}{ }^{0}, i=1, \cdots, m$. These values are required to solve the equations

$$
1+\frac{1}{2} \frac{T_{i}{ }^{1}-2 T_{i}{ }^{0}+T_{i}{ }^{(-1)}}{\left(\Delta y^{2}\right)}=0, \quad i=1, \cdots, m .
$$

10.1. Direct method. Let $U_{i}{ }^{j}$ be the solution of the inhomogeneous equations (10.1), (10.2), where we give the boundary conditions

$$
\begin{array}{ll}
U_{i}^{0}=0, & i=1,2, \cdots, m, \\
U_{m+1}^{j}=U_{0}^{-j}=0, & j=0,1, \cdots, n, \\
U_{i}^{ \pm(n+1)}=\frac{1 \mp x_{i}}{Y}, & i=0,1, \cdots, m+1 .
\end{array}
$$

Let $V_{i}{ }^{j}=V_{i}{ }^{j}(k)$ solve the homogeneous equations formed from (10.1) and (10.2) by omitting the 1 's, and let

$$
\begin{aligned}
& V_{i}^{0}(k)=\delta_{i k}, \\
& V_{m+1}^{j}(k)=V_{0}^{-j}(k)=0, \\
& V_{i}^{ \pm(n+1)}=0,
\end{aligned}
$$$$
i=1,2, \cdots, m \text {, }
$$$$
j=0,1, \cdots, n \text {, }
$$$$
i=0,1, \cdots, m+1 \text {. }
$$

We do this for $k=1, \cdots, m$. 
We now look for $T_{i}{ }^{j}$ as a linear combination

$$
T_{i}{ }^{j}=U_{i}^{j}+\sum_{k=1}^{m} c_{k} V_{i}{ }^{j}(k) .
$$

Every such linear combination satisfies all the conditions required of $T_{i}{ }^{j}$ except possibly the condition (10.3) for $j=0$. The condition (10.3) gives $m$ equations for the $m$ unknowns $c_{1}, \cdots, c_{m}$, which are just the unknown values $T_{1}{ }^{0}, \cdots, T_{m}{ }^{0}$. These equations are nonsingular; otherwise, by the alternative of linear algebra, there would be a solution $H_{i}{ }^{j}$ to the corresponding homogeneous problem with $H_{i}{ }^{0}$ not all zero. But this would violate the maximum principle which holds for the biased difference method.

10.2. Iterative method. We have not used the direct method for any of our calculations, although it looked promising. For both the biased and unbiased methods, we have used iterative schemes. For the biased method, we guess values for $T_{i}{ }^{0}, i=1, \cdots, m$. We then use the equations (10.1) and (10.2) to calculate corresponding values for $T_{i}{ }^{j}$ with $j>0$ and $j<0$. Then (10.3) is used to compute an improved guess:

$$
\text { new } T_{i}{ }^{0}=(\Delta y)^{2}+\left(T_{i}^{1}+T_{i}{ }^{-1}\right) / 2
$$$$
i=1, \cdots, m \text {. }
$$

This process is repeated until some convergence test

$$
\sum_{i=1}^{m}\left(\text { new } T_{i}{ }^{0}-\text { old } T_{i}{ }^{0}\right)^{2}<\epsilon^{2}
$$

is passed.

Now we shall prove the convergence of the iterations. Our linear iterative process is a law of the form

$$
\left(\text { new } T_{i}{ }^{0}\right)=M \cdot\left(\text { old } T_{i}{ }^{0}\right)+f,
$$

where $M$ is some $m \times m$ matrix, and where $f$ is some vector arising from the 1 's in the difference equations and from the nonzero boundary values for $y= \pm Y$. The true value for $T_{i}{ }^{0}$ satisfies

$$
\left(\text { true } T_{i}{ }^{0}\right)=M \cdot\left(\operatorname{true} T_{i}{ }^{0}\right)+f .
$$

Subtracting this equation from the preceding equation, we find

$$
\text { (new error) }=M \cdot(\text { old error }) \text {. }
$$

Letting $v=$ new error, $u=$ old error, we see that $v$ arises from $u$ by an iteration in which all inhomogeneous terms contributing to $f$ are replaced by zero.

Let us, therefore, begin with an initial guess $u_{i}{ }^{0}=u_{i}$. Using the homogeneous difference equations for $j>0$, we compute $u_{i}{ }^{j}$ for $j>0$, and 
similarly we compute $u_{i}^{j}$ for $j<0$. Now the homogeneous equations for $j=0$, coming from (10.3), yield

$$
v_{i}=\left(u_{i}^{1}+u_{i}^{-1}\right) / 2
$$$$
i=1, \cdots, m \text {. }
$$

Assume that the maximum norm $\|u\|=\max \left|u_{i}\right|>0$. By the strong maximum principle for the completely implicit parabolic difference equations for $j>0$, we have the strict inequality

$$
\max \left|u_{i}{ }^{(j)}\right|<\max \left|u_{i}{ }^{0}\right|=\|u\| .
$$

We have the same inequality for $j<0$. Using $j= \pm 1$, we find from (10.13),

$$
\|v\|=\max \left|v_{i}\right|<\left(\max \left|u_{i}\right|+\max \left|u_{i}\right|\right) / 2=\|u\| .
$$

Since $v=M u$, according to (10.12) we have just proved that

$$
\|M\|<1
$$

where $M$ is the regular matrix norm

$$
\|M\|=\max _{u \neq 0} \frac{\|M u\|}{\|u\|}=\max _{u \neq 0} \frac{\|v\|}{\|u\|} .
$$

This proves the convergence of the iterations, since we have after $r$ iterations

$$
\begin{aligned}
(r \text { th error }) & =M^{r} \text { (0th error), } \\
\| r \text { th error } \| & \leqq\|M\|^{r} \| \text { 0th error } \| \rightarrow 0 \text { as } r \rightarrow \infty .
\end{aligned}
$$

11. Solution of the balanced difference equations. If $T_{i+1 / 2}^{0}, i=0, \cdots, m$, were known, the balanced difference method would present a set of CrankNicholson difference equations propagating from right to left where $y$ $>0$, and propagating from left to right where $y<0$. The technique for solving these equations is well known [5, p. 200].

As discussed in the preceding section, a direct method can be employed; but here we shall only discuss an iterative method. We begin with a guess for $T_{i+1 / 2}^{0}, i=0, \cdots, m$. We then use the Crank-Nicholson equations

$$
\begin{aligned}
1+y_{j} \frac{T_{i+1}^{j}-T_{i}{ }^{j}}{\Delta x}+\frac{1}{2} \frac{\delta^{2} T_{i+1 / 2}^{j}}{(\Delta y)^{2}}=0, & \\
j=1,2, \cdots n, \quad & i=0,1, \cdots, m, \\
j=-n, \cdots,-1, & i=0,1, \cdots, m,
\end{aligned}
$$

along with the boundary conditions

$$
\begin{aligned}
T_{m+1}^{j} & =T_{0}^{-j}=0, & j=1, \cdots, n, \\
T_{i+1 / 2}^{ \pm(n+1)} & =\frac{1 \mp x_{i+1 / 2}}{Y}, & i=0, \cdots, m,
\end{aligned}
$$


to compute corresponding values for $T_{i}{ }^{j}$ with $j \neq 0$. We then compute $T_{i}{ }^{0}$ for $i=0, \cdots, m+1$ from the equations

$$
1+\frac{1}{2} \frac{T_{i}^{1}-2 T_{i}^{0}+T_{i}^{-1}}{(\Delta y)^{2}}=0
$$

and from these values for $T_{i}{ }^{0}$ we compute

$$
\text { new } T_{i+1 / 2}^{0}=\left(T_{i}{ }^{0}+T_{i+1 / 2}^{0}\right) / 2, \quad i=0, \cdots, m .
$$

In practice, the last two formulas are replaced by

$\left(11.3^{\prime}\right) \quad$ new $T_{i+1 / 2}^{0}=(\Delta y)^{2}+\left(T_{i+1 / 2}^{1}+T_{i+1 / 2}^{-1}\right) / 2, \quad i=0, \cdots, m$.

This process is repeated until some convergence test

$$
\sum_{i=0}^{m}\left(\text { new } T_{i+1 / 2}^{0}-\text { old } T_{i+1 / 2}^{0}\right)^{2}<\epsilon^{2}
$$

is passed.

The process (11.1), (11.2), (11.3') implicitly defines an $m \times m$ matrix $M$ and an $m$-component vector $f$ such that

$$
\left(\text { new } T_{i+1 / 2}^{0}\right)=M \cdot\left(\text { old } T_{i+1 / 2}^{0}\right)+f,
$$

where $f$ arises from the inhomogeneous terms $1,(1 \mp x) / Y$, and $(\Delta y)^{2}$ in (11.1), (11.2), (11.3'). Setting

$$
\begin{aligned}
\left(\text { new } T_{i+1 / 2}^{0}\right) & =\left(\operatorname{true} T_{i+1 / 2}^{0}\right)+v, \\
\left(\text { old } T_{i+1 / 2}^{0}\right) & =\left(\operatorname{true} T_{i+1 / 2}^{0}\right)+u,
\end{aligned}
$$

we have

$$
v=M u .
$$

Using the norm $\|u\|=\left[\sum\left(u_{i+1 / 2}^{0}\right)^{2}\right]^{1 / 2}$, we shall prove convergence by showing that $\|M\|<1$, where $\|M\|$ is the regular matrix norm

$$
\|M\|=\max _{u \neq 0} \frac{\|M u\|}{\|u\|}=\max _{u \neq 0} \frac{\|v\|}{\|u\|} .
$$

To do this, we only need to show that $\|v\|<\|u\|$ if $\|u\| \neq 0$.

To compute $v$ from $u$, we apply the homogeneous process corresponding to (11.1), (11.2), (11.3'), beginning with $u=u_{i+1 / 2}^{0}$, and ending with $v=v_{i+1 / 2}^{0}$. We first compute numbers $u_{i+1 / 2}^{j}$ for $j \neq 0$ from the homogeneous equations

$$
y_{j} \frac{u_{i+1}^{j}-u_{i}^{j}}{\Delta x}+\frac{1}{2} \frac{\delta^{2} u_{i+1 / 2}^{j}}{(\Delta y)^{2}}=0, \quad|j|=1,2, \cdots, n .
$$


Multiplying these equations by $u_{i+1 / 2}^{j}$, and summing for $i=0, \cdots, m$, we find for $j=1, \cdots, n$,

$$
\frac{y_{j}}{2(\Delta x)}\left[\left(u_{m+1}^{j}\right)^{2}-\left(u_{0}^{j}\right)^{2}\right]+\frac{1}{2(\Delta y)^{2}} \sum_{i=0}^{m} u_{i+1 / 2}^{j} \delta^{2} u_{i+1 / 2}^{j}=0 .
$$

Since $y_{j}>0$ and $u_{m+1}^{j}=0$, we find

$$
\sum_{i=0}^{m} u_{i+1 / 2}^{j} \delta^{2} u_{i+1 / 2}^{j} \geqq 0, \quad j=1,2, \cdots, n .
$$

Summing these inequalities for $j=1, \cdots, n$, and using the homogeneous boundary values $u_{i+1 / 2}^{n+1}=0$, we find

$$
\sum_{i=0}^{m}\left[-u_{i+1 / 2}^{1}\left(u_{i+1 / 2}^{1}-u_{i+1 / 2}^{0}\right)-\sum_{j=1}^{n}\left(u_{i+1 / 2}^{j+1}-u_{i+1 / 2}^{j}\right)^{2}\right] \geqq 0 .
$$

In other words,

$$
\sum_{i=0}^{m}\left(u_{i+1 / 2}^{1}\right)^{2} \leqq \sum_{i=0}^{m} u_{i+1 / 2}^{1} u_{i+1 / 2}^{0}-\sum_{i=0}^{m} \sum_{j=1}^{n}\left(u_{i+1 / 2}^{j+1}-u_{i+1 / 2}^{j}\right)^{2} .
$$

Since $u_{i+1 / 2}^{1} u_{i+1 / 2}^{0} \leqq \frac{1}{2}\left[\left(u_{i+1 / 2}^{1}\right)^{2}+\left(u_{i+1 / 2}^{0}\right)^{2}\right]$, we conclude

$$
\text { (11.10) } \sum_{i=0}^{m}\left(u_{i+1 / 2}^{1}\right)^{2} \leqq \sum_{i=0}^{m}\left(u_{i+1 / 2}^{0}\right)^{2}-2 \sum_{i=0}^{m} \sum_{j=1}^{n}\left(u_{i+1 / 2}^{j+1}-u_{i+1 / 2}^{j}\right)^{2} \text {. }
$$

Using the same reasoning for $j<0$, where $y_{j}<0$ and $u_{0}^{j}=0$, we find

$$
\sum_{i=0}^{m}\left(u_{i+1 / 2}^{-1}\right)^{2} \leqq \sum_{i=0}^{m}\left(u_{i+1 / 2}^{0}\right)^{2}-2 \sum_{i=0}^{m} \sum_{j=-1}^{-n}\left(u_{i+1 / 2}^{j-1}-u_{i+1 / 2}^{j}\right)^{2} .
$$

Since $u_{i+1 / 2}^{n+1}=0$, the double sum $\sum \sum$ in (11.10) is positive unless $u_{i+1 / 2}^{1} \equiv 0$. Therefore, we have the strict inequality

$$
\sum_{i=0}^{m}\left(u_{i+1 / 2}^{1}\right)^{2}<\sum_{i=0}^{m}\left(u_{i+1 / 2}^{0}\right)^{2}
$$

and likewise

$$
\sum_{i=0}^{m}\left(u_{i+1 / 2}^{-1}\right)^{2}<\sum_{i=0}^{m}\left(u_{i+1 / 2}^{0}\right)^{2} .
$$

But from (11.3') we have

$$
v_{i+1 / 2}^{0}=\left(u_{i+1 / 2}^{1}+u_{i+1 / 2}^{-1}\right) / 2, \quad i=0, \cdots, m .
$$

The strict inequalities (11.12) and (11.13) now yield the required result

$$
\|v\|^{2} \equiv \sum_{i=0}^{m}\left(v_{i+1 / 2}^{0}\right)^{2}<\sum_{i=0}^{m}\left(u_{i+1 / 2}^{0}\right)^{2} \equiv\|u\|^{2} .
$$




\section{REFERENCES}

[1] G. Fichera, On a unified theory of boundary value problems for elliptic-parabolic equations of second order, Boundary Problems in Differential Equations, R. E. Langer, ed., University of Wisconsin Press, Madison, 1960, pp. 97-120.

[2] W. H. Fleming, A problem of random accelerations, Tech. Rep. 403, Mathematics Research Center, University of Wisconsin, Madison, 1963.

[3] Handbook of Mathematical F unctions, M. Abramowitz and I. A. Stegun, eds., National Bureau of Standards, Washington, D. C., 1964, Chapters 13 and 15.

[4] K. D. SaKalyuk, Abel's generalized integral equation, Soviet Math. Dokl., 1 (1961), pp. 332-335.

[5] R. D. Richtmyer and K. W. Morton, Difference Methods for Initial-Value Problems, Interscience, New York, 1967.

[6] Selected Papers on Noise and Stochastic Processes, N. Wax, ed., Dover, New York, 1954.

[7] J. J. Kohn and L. Nirenberg, Non-coercive boundary-value problems, Comm. Pure Appl. Math., 18 (1965), pp. 443-492.

[8] O. A. Oleinik, A problem of Fichera, Dokl. Akad. Nauk SSSR, 157 (1964), pp. 1297-1301, Soviet Math. Dokl., 5 (1964), pp. 1129-1133.

[9] J. R. Cannon and C. D. Hill, A finite-difference method for degenerate ellipticparabolic equations, this Journal 5, (1968), pp. 211-218.

[10] G. E. P. Box And Mervin E. Muller, A note on the generation of random normal deviates, Ann. Math. Statist., 29 (1958), pp. 610-611. 